\title{
Synthesis, Biological Evaluation and 2D-QSAR Studies of Novel 6-Oxo-Pyridine-3-Carboxamide Derivatives as Antimicrobial and Antifungal Agents
}

\author{
Hend M. El-Sehrawi ${ }^{1}$, Dalia H.Soliman ${ }^{1}$, Maha M. Khalifa ${ }^{1}$, Omneya M. El-Bakry ${ }^{1}$ \\ ${ }^{1}$ Pharmaceutical Chemistry Department, Faculty of Pharmacy (Girls), Al-Azhar University, Cairo 11754, Egypt \\ Correspondence: Dalia H.Soliman, Pharmaceutical Chemistry Department, Faculty of Pharmacy (Girls), Al-Azhar \\ University, Cairo P.O. Box. 11754, Egypt. Tel: +202-22900776. E-mail: odihss3@gmail.com
}

Received: November 16, 2015 Accepted: December 7, 2015 Online Published: December 18, 2015

doi:10.5539/ijc.v8n1p49

URL: http://dx.doi.org/10.5539/ijc.v8n1p49

\begin{abstract}
In this study twenty six novel derivatives of 6-oxo-pyridine-3-carboxamide were synthesized and evaluated as antibacterial and antifungal agents. Synthesis of the

2-amino-4-methyl-6-oxo-N,1-diphenyl-1,6-dihydropyridine-3-carboxamide 1 was carried out by reacting equimolar quantities of acetoacetanilide and cyanoacetanilide in ethanol using triethylamine as a catalyst. The results of the in vitro antimicrobial evaluation showed that, the

6-oxo-N,1-diphenyl-5-(p-tolyldiazenyl)-1,6-dihydropyridine-3-carboxamide, 5c displayed broad-spectrum antibacterial activity which was equipotent to both Ampicillin and Gentamicin against the tested bacteria. Moreover, compounds 3a, 5c and 9b were equipotent to the reference drug, Amphotericin B, against Aspergillus fumigatus $(\mathrm{MIC}=1.95 \mu \mathrm{g} / \mathrm{ml}) .2 \mathrm{D}$ QSAR studies were carried out in order to correlate the observed activity to the binding mode of these compounds, in addition to their molecular properties that might be controlling their activities.
\end{abstract}

Keywords: synthesis, antimicrobial activity, 6-oxo-pyridine-3-carboxamide; antifungal, 2D-QSAR

\section{Introduction}

In the past few decades, drug-resistant human pathogenic microbes have been developed (Bax, Mullan \& Verhuef, 2000; Cohen, 1992; Finch, Greenwood, Norrby \& Whitley, 2003; Neu, 1998 and Struelens, 1992). The widespread and misuse of antibiotics in humans and animals (Ritter \& Wong, 2001; Alekshun \& Levy, 2007) may directly contribute to this resistance, which in fact, represents a major medical challenge (Setti, Quattrocchio \& Micetich, 1997). Despite the development of several new antibacterial agents, their clinical value is limited in the treatment of an increasing array of life threatening systemic infections. Thus, the development of potent and effective antimicrobial agents is very important to overcome the emerging multi-drug resistance strains of bacteria and fungi. The 2-pyridone has proven to be effective antibacterial and antibacterial agents (Saiki et al, 1999; Li, Mitscher \& Shen, 2000; Gupta \& Plott, 2004; Khokhani, Khatri, \& Patel, 2013; Tipparaju et al, 2008 and Vyas et al, 2008) the clinical candidate, CG400549 (Yum et al, 2007 \& Park et al, 2007) and the promising lead compound, PT171 (Schiebel et al, 2014) were rationally designed as broad spectrum antibacterial agents, figure 1. Moreover, the 2-pyridone CG400549 (Figure 1) was identified as a potent antibacterial against multidrug-resistant staphylococci strains (Kim et al, 2005 \& Gerusz, 2010).

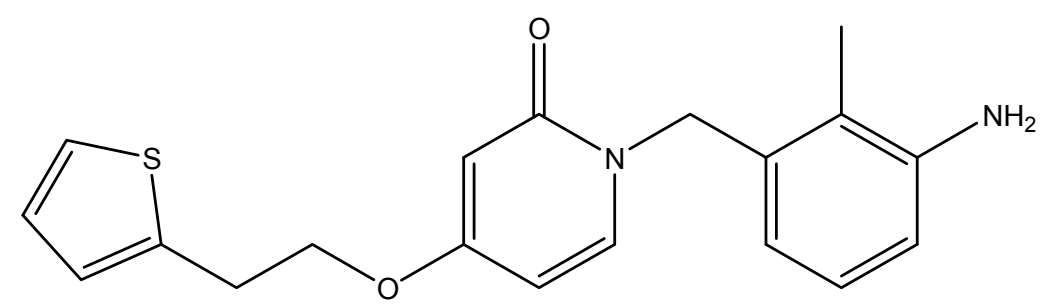

Figure 1. The 2-pyridone based clinical candidate CG400549.

Based on these considerations, we report herein the synthesis, characterization, antibacterial, antifungal and 2D QSAR studies of a novel series of 2-amino-4-methyl-6-oxo-N,1-diphenyl-1,6-dihydropyridine-3-carboxamide, aiming to obtain new potent antibacterial and antifungal agents. A 2D QSAR study was performed on this novel series, whereas, 
the main objective of this study was to develop a relationship between the biological activity of the compounds and their structural or molecular descriptors including, physico-chemical, electronic, geometrical, topological or thermodynamic parameters. Thereby, predicting the activity of novel molecules prior to their synthesis.

\section{Materials and Methods}

\subsection{Experimental}

Melting points were measured with a Stuart melting point apparatus and were uncorrected. The NMR spectra were recorded by Varian Gemini-300BB $300 \mathrm{MHz}$ FT-NMR spectrometers (Varian Inc., Palo Alto, CA). ${ }^{1} \mathrm{H}$ and ${ }^{13} \mathrm{C}$ spectra were run at 300 and $75 \mathrm{MHz}$, respectively, in deuterated dimethylsulphoxide (DMSO- $\left.d_{6}\right)$. Chemical shifts $\left(\delta_{\mathrm{H}}\right)$ are reported relative to TMS as internal standard. All coupling constant $(J)$ values are given in hertz. Chemical shifts $\left(\delta_{\mathrm{C}}\right)$ are reported relative to DMSO- $d_{6}$ as internal standards. The abbreviations used are as follows: $\mathrm{s}$, singlet; $\mathrm{d}$, doublet; $\mathrm{m}$, multiplet. IR spectra were recorded with a Bruker FT-IR spectrophotometer. Electron impact mass spectra were measured on a Varian MAT 311-A (70 e.v.). Reaction courses and product mixtures were routinely monitored by thin layer chromatography (TLC) on silica gel precoated $\mathrm{F}_{254}$ Merck plates. Unless otherwise noted, all solvents and reagents were commercially available and used without further purification.

\section{Synthesis of 2-amino-4-methyl-6-oxo-N,1-diphenyl-1,6-dihydropyridine-3-carboxamide (1)}

Equimolar amounts of acetoacetanilide $(0.012 \mathrm{~mol}, 2.2 \mathrm{gm})$ and cyanoacetanilide $(0.012 \mathrm{~mol}, 2 \mathrm{gm})$ were dissolved in ethanol $(10 \mathrm{ml})$ containing a catalytic amount of triethylamine. The mixture was refluxed for $8 \mathrm{~h}$. The precipitated solid obtained after cooling, was filtered off, washed with ethanol, dried and recrystallized out from ethanol.

Yield (45\%), m.p. $=180-3{ }^{\circ} \mathrm{C} .(\mathrm{EtOH}) ; v\left(\mathrm{~cm}^{-1}\right)(\mathrm{KBr}) 3274,3211\left(\mathrm{NH}, \mathrm{NH}_{2}\right), 1668,1617$ (CO). ${ }^{1} \mathrm{HNMR}$ (DMSO- $\left.\mathrm{d}_{6}-\mathrm{D}_{2} \mathrm{O}\right): \delta 2.20\left(\mathrm{~s}, 3 \mathrm{H}, \mathrm{CH}_{3}\right) ; 3.87\left(\mathrm{~s}, 2 \mathrm{H}, \mathrm{NH}_{2} ; \mathrm{D}_{2} \mathrm{O}\right.$ exchangeable); 7.09-7.11 (t, 2H, 2ph- $\left.\mathrm{H}_{4}\right) ; 7.30-7.35(\mathrm{~m}$, $5 \mathrm{H}, 2 \mathrm{ph}-\mathrm{H}_{3,5}+$ pyridone- $\left.\mathrm{H}\right) ; 7.52\left(\mathrm{~d}, 4 \mathrm{H}, 2 \mathrm{ph}-\mathrm{H}_{2,6}\right) ; 10.23\left(\mathrm{~s}, 1 \mathrm{H}, \mathrm{NH} ; \mathrm{D}_{2} \mathrm{O}\right.$ exchangeable) ${ }^{13} \mathrm{C}-\mathrm{NMR} \delta$, ppm (DMSO-d6): 26.65, 115.83, 119.22, 123.85, 128.84, 138.30, 160.91. Anal. Calcd. for $\mathrm{C}_{19} \mathrm{H}_{17} \mathrm{~N}_{3} \mathrm{O}_{2}$; M.wt: 320; C, 71.46; H, 5.37; N, 13.16; (\%); Found: C, 71.69; H, 5.42; N, 13.39; (\%). MS: m/z (\%): $320\left(\mathrm{M}^{+1}, 19.06\right), 52(100)$.

\section{Synthesis of 2-(benzylideneamino)-4-methyl-6-oxo-N,1-diphenyl-1,6-dihydropyridine-3-carboxamide (2a-f)}

A mixture of 2-amino-1,6-dihydro-4-methyl-6-oxo-N,1-diphenylpyridine-3-carboxamide $\mathbf{1}$ and the appropriate substituted aromatic aldehyde $(0.0015 \mathrm{~mol})$, glacial acetic acid and ethanol was refluxed for $(8 \mathrm{~h} 2 \mathrm{a}-\mathrm{b} / 18 \mathrm{~h} 2 \mathrm{c}-\mathrm{f})$. The precipitated solid formed after cooling was filtered off, washed and recrystallized from ethanol.

2-((4-Chlorobenzylidene)amino)-4-methyl-6-oxo-N,1-diphenyl-1,6-dihydropyridine-3-carboxamide (2a). Yield (67.9\%), m.p. $=208-10{ }^{\circ} \mathrm{C}$; IR (KBr) $v \mathrm{~cm}^{-1}: 3321(\mathrm{NH}), 1675,1594(\mathrm{CO}) .{ }^{1} \mathrm{HNMR}\left(\mathrm{DMSO}^{-} \mathrm{d}_{6}-\mathrm{D}_{2} \mathrm{O}\right): \delta 2.08(\mathrm{~s}, 3 \mathrm{H}$, $\left.\mathrm{CH}_{3}\right)$, 7.12-7.70 (m, 13H, Ar-H + pyridone-H), $7.98\left(\mathrm{~d}, 2 \mathrm{H}, 4-\mathrm{Cl}-\mathrm{ph}-\mathrm{H}_{2,6}\right), 8.27(\mathrm{~s}, 1 \mathrm{H}, \mathrm{N}=\mathrm{CH}), 10.37\left(\mathrm{~s}, 1 \mathrm{H}, \mathrm{NH} ; \mathrm{D}_{2} \mathrm{O}\right.$ exchangeable). Anal. Calcd. for $\mathrm{C}_{26} \mathrm{H}_{20} \mathrm{ClN}_{3} \mathrm{O}_{2}$; Mwt: 441; C, 70.67; H, 4.56; N, 9.51; (\%); Found: C, 70.82; H, 4.61; N, $9.68(\%)$. MS m/z (\%): $443(\mathrm{M}+2,2.46), 441\left(\mathrm{M}^{+}, 1.28\right), 57(100)$.

2-((4-Fluorobenzylidene)amino)-4-methyl-6-oxo-N,1-diphenyl-1,6-dihydropyridine-3-carboxamide (2b). Yield $(45 \%)$, m.p. $=208-11{ }^{\circ} \mathrm{C}$; IR $\left.(\mathrm{KBr}) \vee \mathrm{cm}^{-1}\right) 3315(\mathrm{NH}), 1674,1592(\mathrm{CO}) .{ }^{1} \mathrm{H}-\mathrm{NMR}\left(\mathrm{DMSO}-\mathrm{d}_{6}-\mathrm{D}_{2} \mathrm{O}\right): \delta 2.09(\mathrm{~s}, 3 \mathrm{H}$, $\left.\mathrm{CH}_{3}\right), 7.12-7.68\left(\mathrm{~m}, 13 \mathrm{H}, \mathrm{Ar}-\mathrm{H}+\right.$ pyridone-H), $8.05\left(\mathrm{~d}, 2 \mathrm{H}, 4-\mathrm{F}-\mathrm{ph}-\mathrm{H}_{2,6}\right), 8.28(\mathrm{~s}, 1 \mathrm{H}, \mathrm{N}=\mathrm{C} \underline{\mathrm{H}}), 10.35\left(\mathrm{~s}, 1 \mathrm{H}, \mathrm{NH} ; \mathrm{D}_{2} \mathrm{O}\right.$ exchangeable). Anal. Calcd. for $\mathrm{C}_{26} \mathrm{H}_{20} \mathrm{FN}_{3} \mathrm{O}_{2}$; Mwt: 427; C, 73.40; H, 4.74; N, 9.88; (\%); Found: C, 73.54; H, 4.82; N, 9.94 (\%). MS: $\mathrm{m} / \mathrm{z}(\%): 443\left[\left(\mathrm{M}+\mathrm{H}_{2} \mathrm{O}\right)^{+}, 11.40\right], 174(100)$.

2-((4-Methoxybenzylidene)amino)-4-methyl-6-oxo-N,1-diphenyl-1,6-dihydropyridine-3-carboxamide (2c). Yield $(64.23 \%)$, m.p. $=150-2{ }^{\circ} \mathrm{C} . \mathrm{IR}(\mathrm{KBr}) \vee \mathrm{cm}^{-1}: 3317(\mathrm{NH}), 1674,1591(\mathrm{CO}) .{ }^{1} \mathrm{HNMR}\left(\mathrm{DMSO}-\mathrm{d}_{6}-\mathrm{D}_{2} \mathrm{O}\right): \delta 2.09(\mathrm{~s}, 3 \mathrm{H}$, $\left.\mathrm{CH}_{3}\right), 3.88\left(\mathrm{~s}, 3 \mathrm{H}, \mathrm{OCH}_{3}\right), 7.01-7.39(\mathrm{~m}, 11 \mathrm{H}, \mathrm{ph}-\mathrm{H}+$ pyridone- $\mathrm{H}), 7.65\left(\mathrm{~d}, 2 \mathrm{H}, 4-\mathrm{OCH}_{3}-\mathrm{ph}-\mathrm{H}_{3,5}, J=9 \mathrm{~Hz}\right), 8.0(\mathrm{~d}, 2 \mathrm{H}$, 4- $\left.\mathrm{OCH}_{3}-\mathrm{ph}-\mathrm{H}_{2,6}, J=9 \mathrm{~Hz}\right), 8.20(\mathrm{~s}, 1 \mathrm{H}, \mathrm{N}=\mathrm{CH}), 10.21\left(\mathrm{~s}, 1 \mathrm{H}, \mathrm{NH}\right.$; exchangeable with $\left.\mathrm{D}_{2} \mathrm{O}\right)$. Anal. Calcd. for $\mathrm{C}_{27} \mathrm{H}_{23} \mathrm{~N}_{3} \mathrm{O}_{3}$; Mwt: 437; C, 74.12; H, 5.30; N, 9.60; (\%). Found: C, 74.23; H, 5.36; N, $9.71(\%)$. MS: m/z (\%): $437\left(\mathrm{M}^{+}\right.$, 3.64), 53 (100).

4-Methyl-6-oxo-N,1-diphenyl-2-((3-phenylallylidene)amino)-1,6-dihydropyridine-3-carboxamide (2d). Yield (39.78\%), m.p. $=196-8{ }^{\circ} \mathrm{C}$. IR (KBr) $v \mathrm{~cm}^{-1}: 3346(\mathrm{NH}), 1681,1602(\mathrm{CO}) .{ }^{1} \mathrm{HNMR}\left(\mathrm{DMSO}-\mathrm{d}_{6}-\mathrm{D}_{2} \mathrm{O}\right): \delta 2.50(\mathrm{~s}, 3 \mathrm{H}$, $\left.\mathrm{CH}_{3}\right), 7.13-7.73(\mathrm{~m}, 18 \mathrm{H}, \mathrm{Ar}-\mathrm{H}+$ pyridone- $\mathrm{H}$ and $\mathrm{C} \underline{\mathrm{H}}=\mathrm{C} \underline{\mathrm{H}}), 8.10(\mathrm{~d}, 1 \mathrm{H}, \mathrm{N}=\mathrm{C} \underline{\mathrm{H}}), 10.26(\mathrm{~s}, 1 \mathrm{H}, \mathrm{NH}$; exchangeable with $\mathrm{D}_{2} \mathrm{O}$ ). Anal. Calcd. for $\mathrm{C}_{28} \mathrm{H}_{23} \mathrm{~N}_{3} \mathrm{O}_{2}$; Mwt: 433; C, 77.58; H, 5.35; N, 9.69; (\%). Found: C, 77.64; H, 5.38; N, 9.82 (\%). MS: m/z (\%): $433\left(\mathrm{M}^{+}, 1.79\right), 116(100)$.

4-Methyl-2-((4-nitrobenzylidene)amino)-6-oxo-N,1-diphenyl-1,6-dihydropyridine-3-carboxamide (2e). Yield (36.69\%), m.p. $=208-10{ }^{\circ} \mathrm{C}$. IR $(\mathrm{KBr}) \vee \mathrm{cm}^{-1}: 3324(\mathrm{NH}), 1675,1599(\mathrm{CO}), 1524,1341\left(\mathrm{NO}_{2}\right) .{ }^{1} \mathrm{HNMR}$ (DMSO-d $\left.{ }_{6}-\mathrm{D}_{2} \mathrm{O}\right): \delta 2.48\left(\mathrm{~s}, 3 \mathrm{H}, \mathrm{CH}_{3}\right), 7.14-7.19\left(\mathrm{t}, 2 \mathrm{H}, 2 \mathrm{ph}-\mathrm{H}_{4}\right), 7.35-7.40\left(\mathrm{~m}, 5 \mathrm{H}, 2 \mathrm{ph}-\mathrm{H}_{3,5}+\right.$ pyridone-H), $7.60(\mathrm{~d}, 4 \mathrm{H}$,

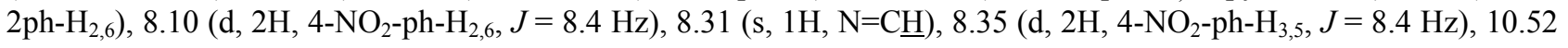


(s,1H, NH; exchangeable with $\mathrm{D}_{2} \mathrm{O}$ ). Anal. Calcd. for $\mathrm{C}_{26} \mathrm{H}_{20} \mathrm{~N}_{4} \mathrm{O}_{4}$; Mwt: 452; C, 69.02; H, 4.46; N, 12.38; (\%). Found: C, 69.09; H, 4.51; N, 12.47 (\%). MS: m/z (\%): $452\left(\mathrm{M}^{+}, 1.54\right), 261$ (100).

4-Methyl-2-((4-methylbenzylidene)amino)-6-oxo-N,1-diphenyl-1,6-dihydropyridine-3-carboxamide (2f). Yield (45.46\%), m.p. $=170-2{ }^{\circ} \mathrm{C} . \mathrm{IR}(\mathrm{KBr}) v \mathrm{~cm}^{-1}: 3337(\mathrm{NH}), 1681,1588(\mathrm{CO}) .{ }^{1} \mathrm{HNMR}\left(\mathrm{DMSO}-\mathrm{d}_{6}-\mathrm{D}_{2} \mathrm{O}\right): \delta 2.15(\mathrm{~s}, 3 \mathrm{H}$, $\left.\mathrm{CH}_{3}\right), 2.40\left(\mathrm{~s}, 3 \mathrm{H}, \mathrm{ph}-\mathrm{CH}_{3}\right), 7.14-7.16\left(\mathrm{~m}, 2 \mathrm{H}, 2 \mathrm{ph}-\mathrm{H}_{4}\right), 7.37-7.43(\mathrm{~m}, 9 \mathrm{H}, \mathrm{ph}-\mathrm{H}+$ pyridone-H), $7.65(\mathrm{~d}, 2 \mathrm{H}$, 4-methyl-ph- $\left.\mathrm{H}_{3,5}, J=9 \mathrm{~Hz}\right), 7.89$ (d, 2H, 4-methyl-ph- $\left.\mathrm{H}_{2,6}, J=9 \mathrm{~Hz}\right), 8.23$ (s, 1H, N=CH) ,10.35(s, 1H, NH; exchangeable with $\mathrm{D}_{2} \mathrm{O}$ ). Anal. Calcd. for $\mathrm{C}_{27} \mathrm{H}_{23} \mathrm{~N}_{3} \mathrm{O}_{2}$; Mwt: 421; C, 76.94; H, 5.50; N, 9.97; (\%). Found: C, 77.08; $\mathrm{H}$, 5.57; N, 10.08 (\%). MS: m/z (\%): 421( $\left.\mathrm{M}^{+}, 0.63\right), 261(100)$.

Synthesis of 2-benzamido-4-methyl-6-oxo-N,1-diphenyl-1,6-dihydropyridine-3-carboxamide (3a-c).

A mixture of 2-amino-1,6-dihydro-4-methyl-6-oxo-N,1-diphenylpyridine-3-carboxamide $\mathbf{1}$ and substituted benzoyl chloride $(0.0015 \mathrm{~mol})$ was refluxed for $10 \mathrm{~h}$. in pyridine. The reaction mixture was poured on ice-water then acidified with $\mathrm{HCl}$. The solid precipitate was filtered off, washed with water, dried and recrystallized from the appropriate solvent.

\section{2-Benzamido-4-methyl-6-oxo-N,1-diphenyl-1,6-dihydropyridine-3-carboxamide (3a).}

Yield (75.4\%), m.p. $=160-2{ }^{\circ} \mathrm{C}$. IR (KBr) $v \mathrm{~cm}^{-1}: 3269,3208(2 \mathrm{NH}), 1666,1613(\mathrm{CO}) .{ }^{1} \mathrm{HNMR}\left(\mathrm{DMSO}-\mathrm{d}_{6}-\mathrm{D}_{2} \mathrm{O}\right): \delta$ $3.36\left(\mathrm{~s}, 3 \mathrm{H}, \mathrm{CH}_{3}\right), 7.06-7.11(\mathrm{~m}, \mathrm{Ar}-\mathrm{H}+$ pyridone-H), 7.30-7.35 (m, Ar- $\mathrm{H}), 7.55\left(\mathrm{~d}, 4 \mathrm{H}, 2 \mathrm{ph}-\mathrm{H}_{2,6}\right), 7.76(\mathrm{~d}, 2 \mathrm{H}$, phenylcarboxamide- $\mathrm{H}_{2,6}$ ), 10.28 (s, $2 \mathrm{H}, 2 \mathrm{NH}$; exchangeable with $\mathrm{D}_{2} \mathrm{O}$ ). Anal. Calcd. for $\mathrm{C}_{26} \mathrm{H}_{21} \mathrm{~N}_{3} \mathrm{O}_{3}$; Mwt: 423; C, 73.74; H, 5.00, N, 9.92; (\%). Found: C, 73.97; H, 5.12; N, 10.06 (\%). MS m/z (\%): 425 (M+2, 0.09), 64 (100).

2-(4-Chlorobenzamido)-4-methyl-6-oxo-N,1-diphenyl-1,6-dihydropyridine-3-carboxamide (3b). Yield (79.57\%); m.p. $=164-6{ }^{\circ} \mathrm{C}$. IR (KBr) $v \mathrm{~cm}^{-1}: 3269,3208(2 \mathrm{NH}), 1665,1612(\mathrm{CO}) .{ }^{1} \mathrm{HNMR}\left(\mathrm{DMSO}-\mathrm{d}_{6}-\mathrm{D}_{2} \mathrm{O}\right) \mathrm{ppm}: \delta 3.38(\mathrm{~s}, 3 \mathrm{H}$, $\mathrm{CH}_{3}$ ), 7.07-7.55 (m, $11 \mathrm{H}$, ph-H and pyridone-H), 7.59 (d, 2H, 4-Cl-ph- $\left.\mathrm{H}_{3,5}, J=8.1 \mathrm{~Hz}\right), 7.97$ (d, 2H,4-Cl-ph- $\mathrm{H}_{2,6}, J=$ 8.1 Hz), 10.27 (s , 2H, 2NH; exchangeable with $\mathrm{D}_{2} \mathrm{O}$ ). Anal. Calcd. for $\mathrm{C}_{26} \mathrm{H}_{20} \mathrm{ClN}_{3} \mathrm{O}_{3}$; Mwt: 459; C, 68.20; $\mathrm{H}, 4.40 ; \mathrm{N}$, 9.18; (\%). Found: C, 68.32; H, 4.45; N, 9.37 (\%). MS m/z (\%): 458 [( $\left.\left(\mathrm{M}^{+2}-1\right)^{+}, 20.64\right], 456\left[(\mathrm{M}-1)^{+}, 24.78\right], 411(100)$.

4-Methyl-2-(4-nitrobenzamido)-6-oxo-N,1-diphenyl-1,6-dihydropyridine-3-carboxamide (3c). Yield (54.5\%), m.p. $=155-7^{\circ} \mathrm{C} . \mathrm{IR}(\mathrm{KBr}) v \mathrm{~cm}^{-1}: 3270,3208(2 \mathrm{NH}), 1664,1603(\mathrm{CO}), 1558,1346\left(\mathrm{NO}_{2}\right) .{ }^{1} \mathrm{HNMR}\left(\mathrm{DMSO}_{6}-\mathrm{d}_{6}-\mathrm{D}_{2} \mathrm{O}\right): \delta 3.34$ (s, 3H, $\left.\mathrm{CH}_{3}\right), 7.09-7.27\left(\mathrm{~m}, 2 \mathrm{H}, 2 \mathrm{ph}-\mathrm{H}_{4}\right), 7.30-7.33\left(\mathrm{~m}, 5 \mathrm{H}, 2 \mathrm{ph}-\mathrm{H}_{3,5}+\right.$ pyridone- $\left.\mathrm{H}\right), 7.52\left(\mathrm{~d}, 4 \mathrm{H}, 2 \mathrm{ph}-\mathrm{H}_{2,6}\right), 8.17(\mathrm{~d}, 2 \mathrm{H}$, 4- $\left.\mathrm{NO}_{2}-\mathrm{ph}-\mathrm{H}_{2,6}, J=8.7 \mathrm{~Hz}\right), 8.36\left(\mathrm{~d}, 2 \mathrm{H}, 4-\mathrm{NO}_{2}-\mathrm{ph}-\mathrm{H}_{3,5}, J=8.7 \mathrm{~Hz}\right), 10.28\left(\mathrm{~s}, 2 \mathrm{H}, 2 \mathrm{NH}\right.$; exchangeable with $\left.\mathrm{D}_{2} \mathrm{O}\right)$. Anal. Calcd. for $\mathrm{C}_{26} \mathrm{H}_{20} \mathrm{~N}_{4} \mathrm{O}_{5}$; Mwt: 466; C, 66.66; H, 4.30; N, 11.96; (\%). Found: C, 66.81; H, 4.36; N, 12.08 (\%). MS m/z (\%): $464\left[\left(\mathrm{M}-2 \mathrm{H}_{2}\right)^{+}, 0.82\right], 92(100)$.

Synthesis of 2-(alkylamino)-4-methyl-6-oxo-N,1-diphenyl-1,6-dihydropyridine-3-carboxamide (4a-b).

Compound 1 (0.0025 mol, $0.79 \mathrm{gm})$ was dissolved in the least amount of DMF then added to a stirred solution of $\mathrm{NaOH}$ $(0.01 \mathrm{~mol}, 0.4 \mathrm{gm})$ in DMF $(10 \mathrm{ml})$. Dimethylsulphate or diethylsulphate $(0.00275 \mathrm{~mol})$ was added drop-wise. The mixture was stirred for $3 \mathrm{~h}$ after which the reaction mixture was poured on ice-water and acidified with $\mathrm{HCl}$. The solid precipitate was filtered off, washed with water, dried and recrystallized from the appropriate solvent.

4-Methyl-2-(methylamino)-6-oxo-N,1-diphenyl-1,6-dihydropyridine-3-carboxamide (4a). Yield (28.73\%), m.p. = $138-40{ }^{\circ} \mathrm{C}$. IR (KBr) $v \mathrm{~cm}^{-1}: 3293(2 \mathrm{NH}), 1689,1600(\mathrm{CO}) .{ }^{1} \mathrm{HNMR}\left(\mathrm{DMSO}_{-} \mathrm{d}_{6}-\mathrm{D}_{2} \mathrm{O}\right): \delta 2.40\left(\mathrm{~s}, 3 \mathrm{H}, \mathrm{CH}_{3}\right), 3.75(\mathrm{~s}, 3 \mathrm{H}$, $\left.\mathrm{N}-\mathrm{CH}_{3}\right), 5.12\left(\mathrm{~s}, 1 \mathrm{H}, \mathrm{NH}\right.$; exchangeable with $\left.\mathrm{D}_{2} \mathrm{O}\right), 7.12-7.64(\mathrm{~m}, 11 \mathrm{H}$, Ar-H + pyridone-H), $9.71(\mathrm{~s}, 1 \mathrm{H}, \mathrm{NH}$; exchangeable with $\mathrm{D}_{2} \mathrm{O}$ ). Anal. Calcd. for $\mathrm{C}_{20} \mathrm{H}_{19} \mathrm{~N}_{3} \mathrm{O}_{2}$; Mwt: 333; C, 72.05; H, 5.74; N, 12.60; (\%). Found: C, 72.19; H, 5.81; N, 12.78 (\%). MS m/z (\%): $333\left[\mathrm{M}^{+}, 45.71\right], 286(100)$.

2-(Ethylamino)-4-methyl-6-oxo-N,1-diphenyl-1,6-dihydropyridine-3-carboxamide (4b). Yield (36.77 \%), m.p. = 150-2 ${ }^{\circ} \mathrm{C}$. IR (KBr) $v \mathrm{~cm}^{-1}: 3299,3191(2 \mathrm{NH}), 1651,1617$ (CO). ${ }^{1} \mathrm{HNMR}$ (DMSO-d ${ }_{6}-\mathrm{D}_{2} \mathrm{O}$ ): $\delta 1.05$ (m, 3H, $\mathrm{NCH}_{2}-\mathrm{CH}_{3}$ ), $1.23\left(\mathrm{~s}, 3 \mathrm{H}, \mathrm{CH}_{3}\right), 3.2\left(\mathrm{~m}, 2 \mathrm{H}, \mathrm{CH}_{2}\right), 7.04-7.64(\mathrm{~m}, 11 \mathrm{H}, \mathrm{Ar}-\mathrm{H}$ and pyridone $-\mathrm{H}), 9.72(\mathrm{~s}, 1 \mathrm{H}, \mathrm{NH}$; exchangeable with $\mathrm{D}_{2} \mathrm{O}$ ), $11.36\left(\mathrm{~s}, 1 \mathrm{H}, \mathrm{NH}\right.$; exchangeable with $\mathrm{D}_{2} \mathrm{O}$ ). Anal. Calcd. for $\mathrm{C}_{21} \mathrm{H}_{21} \mathrm{~N}_{3} \mathrm{O}_{2} ;$ Mwt: 347; C, 72.60; H, 6.09; N, 12.10; (\%). Found: C, 72.68; H, 6.17; N, 12.22 (\%). MS m/z (\%): 347 (M+2.27), 93 (100).

Synthesis

2-amino-4-methyl-6-oxo-N,1-diphenyl-5-((4-substitutedphenyl)diazenyl)-1,6-dihydropyridine-3-carboxamide (5a-d).

A mixture of compound $1(0.0015 \mathrm{~mol}, 0.5 \mathrm{gm})$ and sodium acetate trihydrate $(0.004 \mathrm{~mol}, 0.64 \mathrm{~g})$ in ethanol $(50 \mathrm{ml})$ was stirred at $0^{\circ} \mathrm{C}$. A cold solution of the appropriate diazonium chloride was added drop-wise over a period of $20 \mathrm{~min}$. [prepared by addition of sodium nitrite $(0.12 \mathrm{~g}, 0.001 \mathrm{~mol})$ to appropriate aromatic amine (aniline, p-nitroaniline, p-toluidine and p-anisidine) $(0.0015 \mathrm{~mol})$ in $\mathrm{HCl}$ at $0-5{ }^{\circ} \mathrm{C}$ over a period of $\left.30 \mathrm{~min}\right]$. After complete addition, the reaction mixture was stirred for further $2 \mathrm{hr}$. The resulting solid was filtered off, and recrystallized from appropriate 
solvent.

2-Amino-4-methyl-6-oxo-N,1-diphenyl-5-(phenyldiazenyl)-1,6-dihydropyridine-3-carboxamide(5a). Yield $=(53 \%)$, $\mathrm{mp}=189-92{ }^{\circ} \mathrm{C}$. IR $(\mathrm{KBr}) v \mathrm{~cm}^{-1}: 3324,3237,3140\left(\mathrm{NH}, \mathrm{NH}_{2}\right), 1651,1598(\mathrm{CO}) .{ }^{1} \mathrm{H}-\mathrm{NMR}\left(\mathrm{DMSO}_{-} \mathrm{d}_{6}-\mathrm{D}_{2} \mathrm{O}\right): \delta 2.49(\mathrm{~s}$, $3 \mathrm{H}, \mathrm{CH} 3), 3.89$ (s, 2H, $\mathrm{NH}_{2}$; exchangeable with $\left.\mathrm{D}_{2} \mathrm{O}\right), 7.09$ - $7.73(\mathrm{~m}, 15 \mathrm{H}, \mathrm{Ar}-\mathrm{H}), 9.91(\mathrm{~s}, 1 \mathrm{H}, \mathrm{NH}$; exchangeable with $\mathrm{D}_{2} \mathrm{O}$ ). Anal. Calcd. for $\mathrm{C}_{25} \mathrm{H}_{21} \mathrm{~N}_{5} \mathrm{O}_{2}$; M.wt: 423; C, 70.91; H, 5.00; N, 16.54; (\%); Found: C, 71.08; H, 5.08; N, 16.72 (\%). MS m/z (\%): $425\left[(\mathrm{M}+2)^{+}, 1.10\right], 78(100)$.

2-Amino-4-methyl-5-((4-nitrophenyl)diazenyl)-6-oxo-N,1-diphenyl-1,6-dihydropyridine-3-carboxamide Yield $=(28.6 \%), \mathrm{mp}=164-6^{\circ} \mathrm{C} . \mathrm{IR}(\mathrm{KBr}) v \mathrm{~cm}^{-1}: 3267,3206,3142\left(\mathrm{NH}, \mathrm{NH}_{2}\right), 1662,1599(\mathrm{CO}), 1554,1339\left(\mathrm{NO}_{2}\right)$. ${ }^{1} \mathrm{H}-\mathrm{NMR}\left(\mathrm{DMSO}-\mathrm{d}_{6}-\mathrm{D}_{2} \mathrm{O}\right): \delta 2.53(\mathrm{~s}, 3 \mathrm{H}, \mathrm{CH} 3), 3.30\left(\mathrm{~s}, 2 \mathrm{H}, \mathrm{NH}_{2}\right.$; exchangeable with $\left.\mathrm{D}_{2} \mathrm{O}\right), 7.06-7.55(\mathrm{~m}, 10 \mathrm{H}, \mathrm{Ar}-\mathrm{H})$, $7.68\left(\mathrm{~d}, 2 \mathrm{H}, 4-\mathrm{NO}_{2}-\mathrm{ph}-\mathrm{H}_{2,6}, J=9 \mathrm{~Hz}\right), 8.25\left(\mathrm{~d}, 2 \mathrm{H}\right.$, , 4-NO $\left.\mathrm{NO}_{2}-\mathrm{ph}_{3,5}, J=9 \mathrm{~Hz}\right), 10.25(\mathrm{~s}, 1 \mathrm{H}, \mathrm{NH}$; exchangeable with $\mathrm{D}_{2} \mathrm{O}$ ). Anal. Calcd. for $\mathrm{C}_{25} \mathrm{H}_{20} \mathrm{~N}_{6} \mathrm{O}_{4}$; M.wt: 468; C, 64.10; H, 4.30; N, 17.94; (\%); Found: C, 64.24; H, 4.36; N, 18.09 (\%). MS m/z (\%): 466 [(M-2) $\left.)^{+}, 10.7\right], 103$ (100).

2-Amino-4-methyl-6-oxo-N,1-diphenyl-5-(p-tolyldiazenyl)-1,6-dihydropyridine-3-carboxamide(5c). Yield = $(21.8 \%), \mathrm{mp}=151-3{ }^{\circ} \mathrm{C}$. IR $(\mathrm{KBr}) v \mathrm{~cm}^{-1}: 3343,3188\left(\mathrm{NH}, \mathrm{NH}_{2}\right), 1643,1596(\mathrm{CO}) .{ }^{1} \mathrm{H}-\mathrm{NMR}\left(\mathrm{DMSO}-\mathrm{d}_{6}-\mathrm{D}_{2} \mathrm{O}\right): \delta 2.48$ $\left(\mathrm{s}, 6 \mathrm{H}, 2 \mathrm{CH}_{3}\right), 3.89$ (s, $2 \mathrm{H}, \mathrm{NH}_{2}$; exchangeable with $\left.\mathrm{D}_{2} \mathrm{O}\right), 7.06-7.55$ (m,10H, Ar-H), $7.60\left(\mathrm{~d}, 2 \mathrm{H}, 4-\mathrm{CH}_{3}-\mathrm{ph}_{2} \mathrm{H}_{3,5}, J=9\right.$ $\mathrm{Hz}), 7.68\left(\mathrm{~d}, 2 \mathrm{H}, 4-\mathrm{CH}_{3}-\mathrm{ph}-\mathrm{H}_{2,6}, J=9 \mathrm{~Hz}\right), 9.87\left(\mathrm{~s}, 1 \mathrm{H}, \mathrm{NH}\right.$; exchangeable with $\left.\mathrm{D}_{2} \mathrm{O}\right)$. Anal. Calcd. for $\mathrm{C}_{26} \mathrm{H}_{23} \mathrm{~N}_{5} \mathrm{O}_{2}$; M.wt: 433; C, 71.38; H, 5.30; N, 16.01; (\%); Found: C, 71.53; H, 5.37; N, $16.32(\%)$. MS m/z (\%): 429 [(M-4H $\left.{ }_{2}\right)^{+}$, 61.25], 93 (100).

2-Amino-5-((4-methoxyphenyl)diazenyl)-4-methyl-6-oxo-N,1-diphenyl-1,6-dihydropyridine-3-carboxamide(5d). Yield $=(61.9 \%), \mathrm{mp}=152-154{ }^{\circ} \mathrm{C} . \mathrm{IR}(\mathrm{KBr}) \mathrm{v} \mathrm{cm}^{-1}: 3346,3224,3127\left(\mathrm{NH}, \mathrm{NH}_{2}\right), 1646,1597(\mathrm{CO}) .{ }^{1} \mathrm{H}-\mathrm{NMR}$ $\left(\mathrm{DMSO}_{-} \mathrm{d}_{6}-\mathrm{D}_{2} \mathrm{O}\right): \delta 2.50(\mathrm{~s}, 3 \mathrm{H}, \mathrm{CH} 3), 3.76\left(\mathrm{~s}, 3 \mathrm{H}, \mathrm{OCH}_{3}\right), 6.96\left(\mathrm{~d}, 2 \mathrm{H}, 4-\mathrm{OCH}_{3}-\mathrm{ph}_{-} \mathrm{H}_{3,5}\right), 7.08-7.71(\mathrm{~m}, 12 \mathrm{H}, \mathrm{Ar}-\mathrm{H}$ and 4- $\left.\mathrm{OCH}_{3}-\mathrm{ph}_{2,6}\right), 9.84\left(\mathrm{~s}, \mathrm{H}, \mathrm{NH}\right.$; exchangeable with $\left.\mathrm{D}_{2} \mathrm{O}\right) .{ }^{13} \mathrm{C}-\mathrm{NMR}\left(\mathrm{DMSO}-\mathrm{d}_{6}\right): \delta 10.56,55.31,106.38,111.57$, 114.35, 117.68, 120.88, 123.82, 128.50, 135.57, 138.08, 156.43, and 159.75. Anal. Calcd. for $\mathrm{C}_{26} \mathrm{H}_{23} \mathrm{~N}_{5} \mathrm{O}_{3}$; M.wt: 453; C, 68.86; H, 5.11; N, 15.44; (\%); Found: C, 69.02; H, 5.19; N, 15.69 (\%). MS m/z (\%): 452 [(M-1) $\left.{ }^{+}, 0.02\right], 335$ (100).

Synthesis of 4-methyl-6-oxo-N,1-diphenyl-2-(3-phenylureido)-1,6-dihydropyridine-3-carboxamide (6).

A mixture of compound 1 and phenyl isocyanate $(0.0015 \mathrm{~mol})$ was fused together for $3 \mathrm{~h}$. The reaction mixture was poured on ice-water. The orange precipitate formed was filtered off, washed with petroleum ether then diethyl ether.

Yield $=(94.6 \%), \mathrm{mp}=158-60{ }^{\circ} \mathrm{C} . \mathrm{IR}(\mathrm{KBr}) \vee \mathrm{cm}^{-1}: 3325,3286,3193(3 \mathrm{NH}), 1709,1650,1596(\mathrm{CO}) .{ }^{1} \mathrm{H}-\mathrm{NMR}$ $\left(\right.$ DMSO$\left._{-} \mathrm{d}_{6}-\mathrm{D}_{2} \mathrm{O}\right): \delta 2.38(\mathrm{~s}, 3 \mathrm{H}, \mathrm{CH} 3), 6.93-6.98\left(\mathrm{t}, 3 \mathrm{H}, 3 \mathrm{ph}-\mathrm{H}_{4}\right), 7.24-7.29\left(\mathrm{t}, 7 \mathrm{H}, 3 \mathrm{ph}-\mathrm{H}_{3,5}+\right.$ pyridone- $\left.\mathrm{H}\right), 7.45(\mathrm{~d}, 6 \mathrm{H}$, 3ph- $\left.\mathrm{H}_{2,6}\right), 8.62\left(\mathrm{~s}, 2 \mathrm{H}, \mathrm{NH}\right.$; exchangeable with $\left.\mathrm{D}_{2} \mathrm{O}\right), 9.69$ (s, $1 \mathrm{H}$, amide- $\mathrm{NH} ; \mathrm{D}_{2} \mathrm{O}$ exchangeable). Anal. Calcd. for $\mathrm{C}_{26} \mathrm{H}_{22} \mathrm{~N}_{4} \mathrm{O}_{3}$; M.wt: 438; C, 71.22; H, 5.06; N, 12.78; (\%). Found: C, 71.34; H, 5.13; N, 12.95 (\%). MS m/z (\%0: 438 $\left[\mathrm{M}^{+}, 6.71\right], 93(100)$.

Synthesis of 2-amino-6-oxo-N,1-diphenyl-4-(4- Substituted styryl)-1,6-dihydropyridine-3-carboxamide (7a-c).

A mixture of equimolar amounts of compound $\mathbf{1}$, the appropriate aromatic aldehyde $(0.0015 \mathrm{~mol})$ in ethanol $(10 \mathrm{ml})$ and a catalytic amount of piperidine was refluxed for $20 \mathrm{~h}$. The precipitated solid formed after cooling was filtered off, washed and recrystallized from ethanol.

2-Amino-4-(4-chlorostyryl)-6-oxo-N,1-diphenyl-1,6-dihydropyridine-3-carboxamide (7a). Yield = $(54.9 \%), \mathrm{mp}=$ 288-90 ${ }^{\circ} \mathrm{C}$. IR $(\mathrm{KBr}) \vee \mathrm{cm}^{-1}: 3296,3224\left(\mathrm{NH}, \mathrm{NH}_{2}\right), 1638,1593(\mathrm{CO}) .{ }^{1} \mathrm{H}-\mathrm{NMR}\left(\mathrm{DMSO}-\mathrm{d}_{6}-\mathrm{D}_{2} \mathrm{O}\right): \delta 6.93(\mathrm{~d}, 1 \mathrm{H}$, $\mathrm{C} \underline{\mathrm{H}}=\mathrm{CH}-4-\mathrm{Cl}-\mathrm{ph}, J=7.8 \mathrm{~Hz}), 7.12-7.39$ (m, 15H, Ar-H + pyridone-H), 7.66 (d, 1H, CH=C $\underline{H}-4-\mathrm{Cl}-\mathrm{ph}, J=7.8 \mathrm{~Hz}), 9.02$ (s, $1 \mathrm{H}, \mathrm{NH}$; exchangeable with $\mathrm{D}_{2} \mathrm{O}$ ). Anal. Calcd. for $\mathrm{C}_{26} \mathrm{H}_{20} \mathrm{ClN}_{3} \mathrm{O}_{2}$; M.wt: 441: C, 70.67; H, 4.56; N, 9.51; (\%). Found: C, 70.89; H, 4.62; N, 9.67 (\%). MS m/z (\%): 443 [(M+2) $\left.{ }^{+}, 1.79\right], 441\left(\mathrm{M}^{+}, 7.25\right), 94(100)$.

2-Amino-4-(4-fluorostyryl)-6-oxo-N,1-diphenyl-1,6-dihydropyridine-3-carboxamide (7b). Yield $=(25.5 \%), \mathrm{mp}=$ 278-80 ${ }^{\circ} \mathrm{C}$. IR (KBr) $v \mathrm{~cm}^{-1}: 3268,3230,3192\left(\mathrm{NH}, \mathrm{NH}_{2}\right), 1640,1594(\mathrm{CO}) .{ }^{1} \mathrm{H}-\mathrm{NMR}\left(\mathrm{DMSO}_{6}-\mathrm{d}_{6}-\mathrm{D}_{2} \mathrm{O}\right): 86.91-7.45$ (m, 16H, Ar-H and $\mathrm{C} \underline{H}=\mathrm{CH}-4-\mathrm{F}-\mathrm{ph}+$ pyridone-H), 7.63 (d, 1H, CH=Cㅍ-4-F-ph), 9.03 (s, 1H, NH; exchangeable with $\mathrm{D}_{2} \mathrm{O}$ ). Anal. Calcd. for $\mathrm{C}_{26} \mathrm{H}_{20} \mathrm{FN}_{3} \mathrm{O}_{2}$; M.wt: 425; C, 73.40; H, 4.74; N, 9.88; (\%). Found: C, 73.59; H, 4.78; N, 9.95 (\%). MS m/z (\%): $425\left(\mathrm{M}^{+}, 18.86\right), 359(100)$.

2-Amino-4-(4-nitrostyryl)-6-oxo-N,1-diphenyl-1,6-dihydropyridine-3-carboxamide (7c). Yield = (66\%), $\mathrm{mp}=$ 118-20 ${ }^{\circ} \mathrm{C}$. IR (KBr) $v \mathrm{~cm}^{-1}: 3349,3264\left(\mathrm{NH}, \mathrm{NH}_{2}\right), 1655,1624$ (CO), 1526, $1350\left(\mathrm{NO}_{2}\right) .{ }^{1} \mathrm{H}-\mathrm{NMR}\left(\mathrm{DMSO}-\mathrm{d}_{6}-\mathrm{D}_{2} \mathrm{O}\right): \delta$ ppm: 6.99-7.60 (m, 13H, Ar- $\mathrm{H}$, pyridone $-\mathrm{H}+$ olefinic protons), $7.68\left(\mathrm{~d}, 2 \mathrm{H}, 4-\mathrm{NO}_{2}-\mathrm{ph}-\mathrm{H}_{2,6}, J=8.4 \mathrm{~Hz}\right), 8.27(\mathrm{~d}, 2 \mathrm{H}$, 4- $\mathrm{NO}_{2}-$ ph- $\left.\mathrm{H}_{3,5}, J=8.4 \mathrm{~Hz}\right), 9.11\left(\mathrm{~s}, 1 \mathrm{H}, \mathrm{NH}\right.$; exchangeable with $\left.\mathrm{D}_{2} \mathrm{O}\right), 10.13\left(\mathrm{~s}, 2 \mathrm{H}, \mathrm{NH} 2\right.$; exchangeable with $\mathrm{D}_{2} \mathrm{O}$ ). Anal. Calcd. for $\mathrm{C}_{26} \mathrm{H}_{20} \mathrm{~N}_{4} \mathrm{O}_{4}$; M.wt: 452; C, 69.02; H, 4.46; N, 12.38; (\%). Found: C, 69.23; H, 4.51; N, 12.46 (\%). MS $\mathrm{m} / \mathrm{z}(\%): 452\left(\mathrm{M}^{+}, 10.67\right), 93(100)$. 
Synthesis

of

2-(((dimethylamino)methylene)amino)-4-methyl-6-oxo-N,1-diphenyl-1,6-dihydropyridine-3-carboxamide (8).

A mixture of equimolar amounts of compound 1and DMF-DMA $(0.0015 \mathrm{~mol})$ in xylene $(5 \mathrm{ml})$ was refluxed for $4 \mathrm{~h}$. The precipitated solid formed after cooling was filtered off and washed with diethyl ether.

Yield $=(88.7 \%), \mathrm{mp}=140-2{ }^{\circ} \mathrm{C} . \mathrm{IR}(\mathrm{KBr}) v \mathrm{~cm}^{-1}: 3327(\mathrm{NH}), 1669,1623(\mathrm{CO}) .{ }^{1} \mathrm{H}-\mathrm{NMR}\left(\mathrm{DMSO}-\mathrm{d}_{6}-\mathrm{D}_{2} \mathrm{O}\right): \delta 2.49(\mathrm{~s}$, $\left.3 \mathrm{H}, \mathrm{CH}_{3}\right), 3.17,3.24\left(2 \mathrm{~s}, 6 \mathrm{H}, \mathrm{N}\left(\mathrm{CH}_{3}\right)_{2}\right), 7.01\left(\mathrm{t}, 2 \mathrm{H}, 2 \mathrm{ph}-\mathrm{H}_{4}\right), 7.26\left(\mathrm{~m}, 5 \mathrm{H}, 2 \mathrm{ph}-\mathrm{H}_{3,5}+\right.$ pyridone $\left.-\mathrm{H}\right), 7.57(\mathrm{~d}, 4 \mathrm{H}$, 2ph- $\left.\mathrm{H}_{2,6}\right), 7.81(\mathrm{~s}, 1 \mathrm{H}, \mathrm{N}=\mathrm{CH}), 9.01\left(\mathrm{~s}, 1 \mathrm{H}, \mathrm{NH}\right.$; exchangeable with $\left.\mathrm{D}_{2} \mathrm{O}\right)$. Anal. Calcd for $\mathrm{C}_{22} \mathrm{H}_{22} \mathrm{~N}_{4} \mathrm{O}_{2} ; \mathrm{Mwt}: 374 ; \mathrm{C}$, 70.57; H, 5.92; N, 14.96; (\%). Found: C, 70.74; H, 5.95; N, 15.21 (\%). MS m/z (\%): $374\left(\mathrm{M}^{+}, 0.09\right), 124$ (100).

\section{Synthesis of compounds $9 \mathrm{a}-\mathrm{c}$}

A solution of compound $8(0.0015 \mathrm{~mol}, 0.5 \mathrm{gm})$ in ethanol $(10 \mathrm{ml})$ was refluxed for $20 \mathrm{~h}$. with an equimolar amount of the appropriate secondary amine. The precipitated solid formed after cooling was filtered off, washed and recrystallized from ethanol.

4-Methyl-2-((morpholinomethylene)amino)-6-oxo-N,1-diphenyl-1,6-dihydropyridine-3-carboxamide (9a). Yield = $(61 \%), \mathrm{mp}>300{ }^{\circ} \mathrm{C}$. IR (KBr) $v \mathrm{~cm}^{-1}: 3332(\mathrm{NH}), 1681,1635(\mathrm{CO}) .{ }^{1} \mathrm{H}-\mathrm{NMR}\left(\mathrm{DMSO}-\mathrm{d}_{6}-\mathrm{D}_{2} \mathrm{O}\right): \delta 2.44(\mathrm{~s}, 3 \mathrm{H}, \mathrm{CH} 3)$, $2.55\left(\mathrm{~m}, 4 \mathrm{H}\right.$, morpholine- $\left.\mathrm{H}_{2,6}\right), 3.35\left(\mathrm{~m}, 4 \mathrm{H}\right.$, morpholine- $\left.\mathrm{H}_{3,5}\right), 7.07-7.62(\mathrm{~m}, 11 \mathrm{H}$, Ar- $\mathrm{H}+$ pyridone- $\mathrm{H}), 8.68(\mathrm{~s}, 1 \mathrm{H}$, $\mathrm{N}=\mathrm{C} \underline{\mathrm{H}}), 9.91\left(\mathrm{~s}, 1 \mathrm{H}, \mathrm{NH}\right.$; exchangeable with $\left.\mathrm{D}_{2} \mathrm{O}\right)$. Anal. Calcd. for $\mathrm{C}_{24} \mathrm{H}_{24} \mathrm{~N}_{4} \mathrm{O}_{3}$; M.wt: 416; C, 69.21; H, 5.81; $\mathrm{N}$, 13.45; (\%). Found: C, 69.29; H, 5.89; N, 13.62 (\%). MS m/z (\%): $416\left(\mathrm{M}^{+}, 1.01\right), 93$ (100).

4-Methyl-6-oxo-N,1-diphenyl-2-((piperazin-1-ylmethylene)amino)-1,6-dihydropyridine-3-carboxamide

(9b). Yield $=(36 \%), \mathrm{mp}>300{ }^{\circ} \mathrm{C}$. IR $(\mathrm{KBr}) v \mathrm{~cm}^{-1}: 3359,3332(2 \mathrm{NH}), 1681,1635(\mathrm{CO}) .{ }^{1} \mathrm{H}-\mathrm{NMR}\left(\mathrm{DMSO}-\mathrm{d}_{6}-\mathrm{D}_{2} \mathrm{O}\right): \delta 2.37$ $\left(\mathrm{s}, 3 \mathrm{H}, \mathrm{CH}_{3}\right), 3.45\left(\mathrm{~m}, 8 \mathrm{H}\right.$, piperazine-H), $4.36\left(\mathrm{~s}, 1 \mathrm{H}, \mathrm{NH}\right.$; exchangeable with $\left.\mathrm{D}_{2} \mathrm{O}\right), 7.06-7.11\left(\mathrm{t}, 2 \mathrm{H}, 2 \mathrm{ph}-\mathrm{H}_{4}\right), 7.31(\mathrm{~s}$, $1 \mathrm{H}$, pyridone-H), 7.33-7.38 (m, 4H, 2ph- $\left.\mathrm{H}_{3,5}\right), 7.56-7.62\left(\mathrm{~d}, 4 \mathrm{H}, 2 \mathrm{ph}-\mathrm{H}_{2,6}\right), 8.68(\mathrm{~s}, 1 \mathrm{H}, \mathrm{N}=\mathrm{CH}), 9.94(\mathrm{~s}, 1 \mathrm{H}, \mathrm{NH}$; exchangeable with $\mathrm{D}_{2} \mathrm{O}$ ). Anal. Calcd. for $\mathrm{C}_{24} \mathrm{H}_{25} \mathrm{~N}_{5} \mathrm{O}_{2}$; M.wt: 415; C, 69.38; H, 6.06; N, 16.86; (\%). Found: C, 69.61; H, 6.12; N, 17.04 (\%). MS m/z (\%): $415\left(\mathrm{M}^{+}, 1.40\right), 93$ (100).

4-Methyl-6-oxo-N,1-diphenyl-2-((piperidin-1-ylmethylene)amino)-1,6-dihydropyridine-3-carboxamide (9c). Yield $=(22.5 \%), \mathrm{mp}=90-2{ }^{\circ} \mathrm{C} . \mathrm{IR}(\mathrm{KBr}) \mathrm{c} \mathrm{cm}^{-1}: 3328(\mathrm{NH}), 1654,1620(\mathrm{CO}) .{ }^{1} \mathrm{H}-\mathrm{NMR}\left(\mathrm{DMSO}-\mathrm{d}_{6}-\mathrm{D}_{2} \mathrm{O}\right): \delta 1.55(\mathrm{~m}, 2 \mathrm{H}$, piperidine- $\left.\mathrm{H}_{4}\right), 2.39\left(\mathrm{~s}, 3 \mathrm{H}, \mathrm{CH}_{3}\right), 2.99\left(\mathrm{~m}, 4 \mathrm{H}\right.$, piperidine- $\left.\mathrm{H}_{3,5}\right), 3.48\left(\mathrm{~m}, 4 \mathrm{H}\right.$, piperidine- $\left.\mathrm{H}_{2,6}\right), 6.90-7.62(\mathrm{~m}, 11 \mathrm{H}, \mathrm{Ar}-\mathrm{H}$

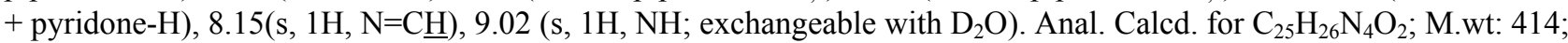
C, 72.44; H, 6.32; N, 13.52; (\%). Found: C, 72.68; H, 6.39; N, 13.78 (\%). MS m/z (\%): 413[(M-1) $\left.)^{+}, 5.44\right], 64(100)$.

Synthesis of compounds $10 a, b$.

A solution of compound $\mathbf{8}(0.0015 \mathrm{~mol}, 0.5 \mathrm{gm})$ in ethanol $(10 \mathrm{ml})$ was refluxed for $20 \mathrm{~h}$ with the appropriate substituted secondary amine. The precipitated solid formed after cooling was filtered off, washed and recrystallized ethanol.

4-Methyl-6-oxo-N,1-diphenyl-2-(((4-phenylpiperazin-1-yl)methylene)amino)-1,6-dihydropyridine-3-carboxamide (10a). Yield $=(38 \%), \mathrm{mp}=185-7{ }^{\circ} \mathrm{C} . \mathrm{IR}(\mathrm{KBr}) \vee \mathrm{cm}^{-1}: 3371(\mathrm{NH}), 1670,1619(\mathrm{CO}) .{ }^{1} \mathrm{H}-\mathrm{NMR}\left(\mathrm{DMSO}-\mathrm{d}_{6}-\mathrm{D}_{2} \mathrm{O}\right): \delta \mathrm{ppm}$ : 2.50 (s, 3H, CH3), $3.15\left(\mathrm{~m}, 4 \mathrm{H}\right.$, piperazine- $\left.\mathrm{H}_{2,6}\right), 3.42\left(\mathrm{~m}, 4 \mathrm{H}\right.$, piperazine- $\left.\mathrm{H}_{3,5}\right), 6.80-7.58(\mathrm{~m}, 16 \mathrm{H}$, Ar- $\mathrm{H}+$ pyridone- $\mathrm{H})$, 7.93 (s, 1H, N=C프), 9.12 (s, 1H, NH; exchangeable with $\mathrm{D}_{2} \mathrm{O}$ ). Anal. Calcd. for $\mathrm{C}_{30} \mathrm{H}_{29} \mathrm{~N}_{5} \mathrm{O}_{2}$; M.wt: 491; C, 73.30; H, 5.95; N, 14.25; (\%). Found: C, 73.47; H, 6.02; N, 14.37 (\%). MS m/z (\%): 493 [(M+2), 2.56$], 104$ (100).

4-Methyl-2-(((4-methylpiperazin-1-yl)methylene)amino)-6-oxo-N,1-diphenyl-1,6-dihydropyridine-3-carboxamide (10b). Yield $=(31.3 \%), \mathrm{mp}>300{ }^{\circ} \mathrm{C} . \mathrm{IR}(\mathrm{KBr}) v \mathrm{~cm}^{-1}: 3332(\mathrm{NH}), 1681,1635(\mathrm{CO}) .{ }^{1} \mathrm{H}-\mathrm{NMR}\left(\mathrm{DMSO}-\mathrm{d}_{6}-\mathrm{D}_{2} \mathrm{O}\right): \delta 2.22$ $(\mathrm{s}, 3 \mathrm{H}, \mathrm{CH} 3), 2.44\left(\mathrm{~s}, 3 \mathrm{H}, \mathrm{N}-\mathrm{CH}_{3}\right), 3.15\left(\mathrm{~m}, 4 \mathrm{H}\right.$, piperazine- $\left.\mathrm{H}_{3,5}\right), 3.42\left(\mathrm{~m}, 4 \mathrm{H}\right.$, piperazine- $\left.\mathrm{H}_{2,6}\right), 7.07-7.12(\mathrm{t}, 2 \mathrm{H}$, 2ph- $\left.\mathrm{H}_{4}\right), 7.31\left(\mathrm{~s}, 1 \mathrm{H}\right.$, pyridone-H), 7.34-7.38 (t, 4H, 2ph- $\left.\mathrm{H}_{3,5}\right), 7.60\left(\mathrm{~d}, 4 \mathrm{H}, 2 \mathrm{ph}-\mathrm{H}_{2,6}\right), 8.69(\mathrm{~s}, 1 \mathrm{H}, \mathrm{N}=\mathrm{C} \underline{\mathrm{H}}), 9.91(\mathrm{~s}, 1 \mathrm{H}$, $\mathrm{NH}$; exchangeable with $\left.\mathrm{D}_{2} \mathrm{O}\right) .{ }^{13} \mathrm{C}-\mathrm{NMR}\left(\mathrm{DMSO}_{6} \mathrm{~d}_{6}\right): \delta 35.91,65.98,66.06,85.02,120.89,126.31,128.50,130.30$, 133.87, 138.51, 146.14, 157.14, and 164.80. Anal. Calcd. for $\mathrm{C}_{25} \mathrm{H}_{27} \mathrm{~N}_{5} \mathrm{O}_{2}$; M.wt: 429; C, 69.91; H, 6.34; N, 16.31; (\%). Found: C, 70.08; H, 6.38; N, 16.58 (\%). MS m/z (\%): $429\left(\mathrm{M}^{+}, 1.38\right), 93$ (100).

\subsection{Antimicrobial Screening}

\section{Methodology of the antimicrobial screening}

The newly synthesized compounds were tested for their antibacterial and antifungal activities according to The Clinical and Laboratory Standards Institute (CLSI), formerly the National Committee for Clinical Laboratory Standards (NCCLS), based on the brothmicrodilution reference method according to the National Committee for Clinical Laboratory Standards (2002).

These assays were performed at the Regional Center for Mycology and Bio-technology, Antimicrobial unit test organisms, Cairo, Egypt. 


\section{Microorganism's strains and preparation of inoculum}

A. fumigatus (RCMB 02568), C. albicans (RCMB 05036), S. pneumoniae (RCMB 010010), B. subtilis (RCMB 010067), P. aeruginosa (RCMB 010049), E. coli (RCMB 010058) and S.Typhimurium (RCMB 010315) Strains were used in this study. The microbial suspension equivalent to the turbidity of $0.5 \mathrm{McFarland}\left(10^{8} \mathrm{CFU} / \mathrm{ml}\right.$ i.e colony forming unit per millilitre) standard was prepared from a fresh subculture of tested bacteria in a Mueller Hinton broth (MHB) and tested with fungi in a Sabouraud dextrose broth (SDB) then this suspension was diluted to $10^{6} \mathrm{CFU} / \mathrm{ml}$ using MHB for bacteria and Sabouraud dextrose Broth (SDB) for tested fungi. The adjusted microbial inoculum $(100 \mu \mathrm{l})$ was added to each well of a sterile 96-well flat-bottomed microtiter plate containing the tested concentration of tested samples (100 $\mu 1 /$ well). Three wells containing a microbial suspension with no sample using DMSO employed for dissolving the tested compound (growth control) and two wells containing only media (background control) were included in this plate. Optical densities were measured after 24 hours at $37^{\circ} \mathrm{C}$ for bacteria and after 48 hours at $28^{\circ} \mathrm{C}$ for fungi using a multi-detection microplate reader at The Regional Center for Mycology and Biotechnology (Sun Rise-Tecan, USA) at $600 \mathrm{~nm}$. Ampicillin, Gentamicin and Amphotericin B were used as standards for Gram positive bacteria, Gram negative bacteria and fungi, respectively. The percentage of microbial inhibitory was calculated using the Microsoft Excel®.

Microbial viability $\%$ was calculated according to the following equation:

$$
\text { Percentage of viability }=[1-(\mathrm{ODt} / \mathrm{ODc})] \times 100 \%
$$

Where, ODt is the mean optical density of wells treated with the tested compound and ODc is the mean optical density of untreated cells, while Inhibitory $\%=(100-$ viability $) \%$.

\section{Minimum Inhibitory Concentration (MIC) \& Inhibitory concentration50 $\left(I C_{50}\right)$ measurement}

For the determination of MIC of tested samples microdilution test was performed in 96-well plates. Two-fold dilutions of each compound were prepared in the test wells, the final drug concentrations being (125-0.004) $\mu \mathrm{g} / \mathrm{mL}$, control wells were prepared with culture medium only and microbial suspension only. The plates were sealed and incubated for 24 hours at $37^{\circ} \mathrm{C}$ for bacteria and for 48 hours at $28^{\circ} \mathrm{C}$ for fungi, after each incubation time. MIC was detected as the lowest sample concentration that prevented microbial growth. Each MIC was determined three times.The test compounds were also compared using the $\mathrm{IC}_{50}$ value, i.e., the concentration of the compound leading to $50 \%$ microbial death that was estimated from graphical plots.

\section{Results and Discussion}

\subsection{Chemistry}

In the present work, synthesis of the novel twenty six 2-pyridone derivatives bearing different aryl and heteroaryl rings are depicted in (Schemes 1-3). Cyanoacetamides are highly reactive compounds that have been extensively utilized in building different organic heterocycles. Thus, the known intermediate cyanoacetanilide was obtained through the fusion of aniline with slight excess of ethyl cyanoacetate (Fadda, Bondock, Rabie \& Etman, 2008). Synthesis of the 2-amino-4-methyl-6-oxo-N,1-diphenyl-1,6-dihydropyridine-3-carboxamide 1 was carried out by reacting equimolar quantities of acetoacetanilide and cyanoacetanilide in ethanol using triethylamine as a catalyst (Fadda, Bondock, Rabie \& Etman, 2008; Ammar et al, 2005; Mohamed, Awad, El-Hallouty \& El-Araby, 2012). 


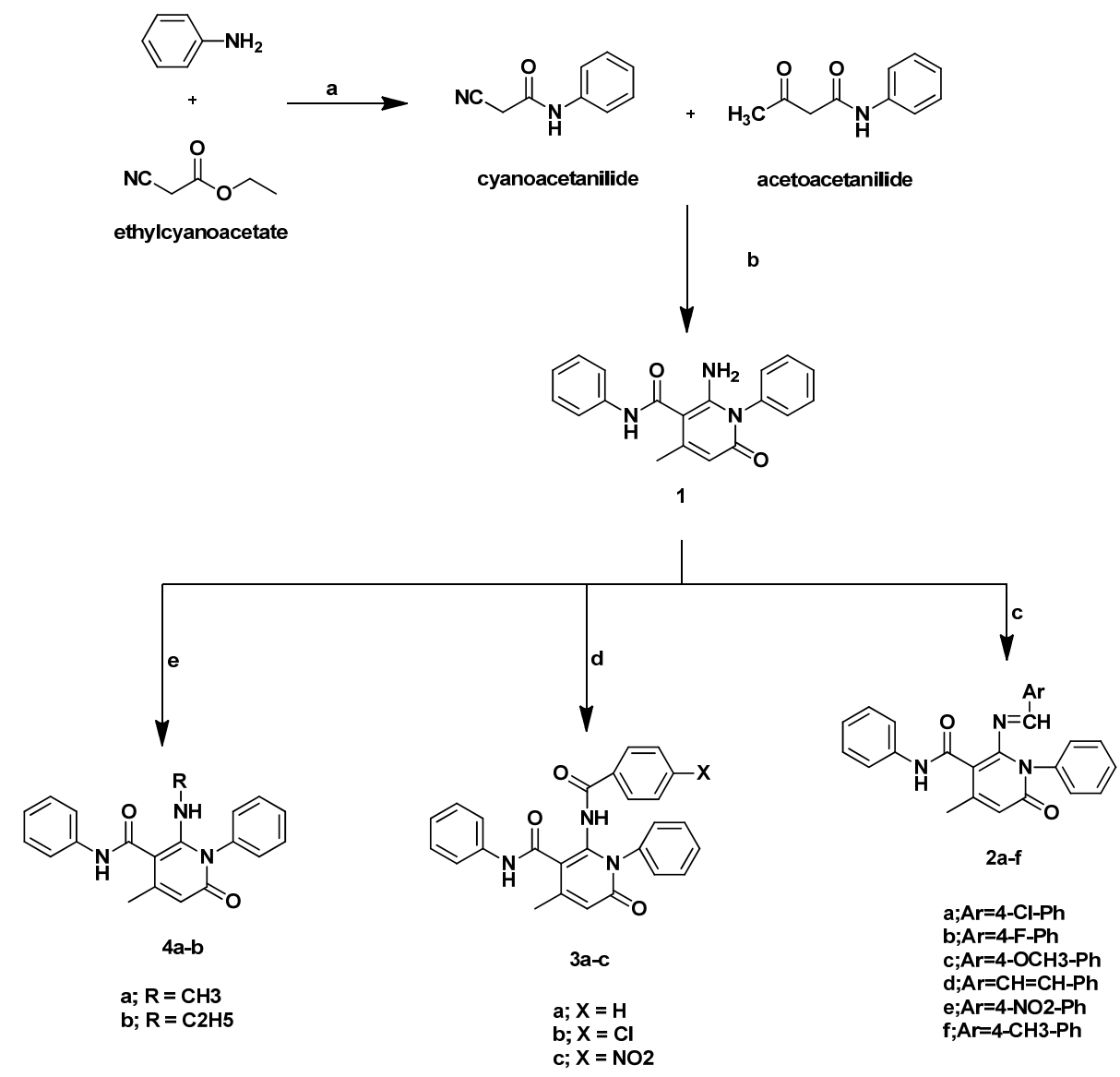

Scheme 1.

Reagents and conditions: a) fusion. b) ethanol, TEA, reflux. c) substituted aromatic aldehydes, glacial acetic acid, ethanol, reflux. d) 4-substituted benzoyl chloride, pyridine, reflux. e) DMS/DES, DMF, $\mathrm{NaOH}$, stirring.

${ }^{1} \mathrm{HNMR}$ spectrum revealed the existence of a singlet at $\delta 2.20 \mathrm{ppm}$ corresponding to $\mathrm{CH}_{3}$ group and two $\mathrm{D}_{2} \mathrm{O}$ exchangeable singlets at $\delta 3.87$ and 10.23 ppm corresponding to $\mathrm{NH}, \mathrm{NH}_{2}$ groups. ${ }^{13} \mathbf{C N M R}$ spectrum revealed the existence of $\mathrm{CH}_{3}$ group at $\delta 26.65 \mathrm{ppm}$ and 2 carbonyl groups at $\delta 160.91 \mathrm{ppm}$ and was taken as an evidence for the formation of the pyridone ring. In order to examine the effect of incorporation of arylideneamino moiety, compounds 2a-f were synthesized via refluxing compound $\mathbf{1}$ with substituted aromatic aldehydes, using absolute ethanol as a solvent and in the presence of catalytic amount of acetic acid (Parmar et al, 2011).

IR spectra of these derivatives exhibited a sharp band around $3321 \mathrm{~cm}^{-1}$ corresponding to NH group along with the disappearance of $\mathrm{NH}_{2}$ bands. Moreover, ${ }^{1} \mathbf{H N M R}$ spectra for this group exhibited a singlet corresponding to the imine proton $(\mathrm{CH}=\mathrm{N})$ at the range $\delta 8.10$ to $8.31 \mathrm{ppm}$ denoting the formation of the schiff's base. Compounds 3a-c were successfully prepared by refluxing equimolar quantities of 1 and substituted benzoyl chloride in pyridine. IR spectra for this series were in agreement with the predicted structures as they showed the disappearance of $\mathrm{NH}_{2}$ bands. In addition, ${ }^{1} \mathbf{H N M R}$ spectra of $\mathbf{3 b}$ and $\mathbf{3 c}$, as representatives of this group,displayed two doublets corresponding to the para-substituted systems at $\delta 7.59,7.97 \mathrm{ppm}$ and at $\delta 8.17,8.36 \mathrm{ppm}$ with $J$ constant $=8.1$ and $8.7 \mathrm{~Hz}$ respectively. Alkylation of the amino group was achieved by the reaction of compound $\mathbf{1}$ with dimethylsulfate or diethylsulfate in DMF / $\mathrm{NaOH}$ to afford compounds $\mathbf{4 a , b} .{ }^{1} \mathbf{H N M R}$ spectrum of compound 4a showed a singlet at $\delta 3.75 \mathrm{ppm}$ assigned to the methyl group, as for compound $\mathbf{4 b}$, the triplet quartet pattern of the N-ethyl group appeared at $\delta 1.05$ and $3.20 \mathrm{ppm}$. 


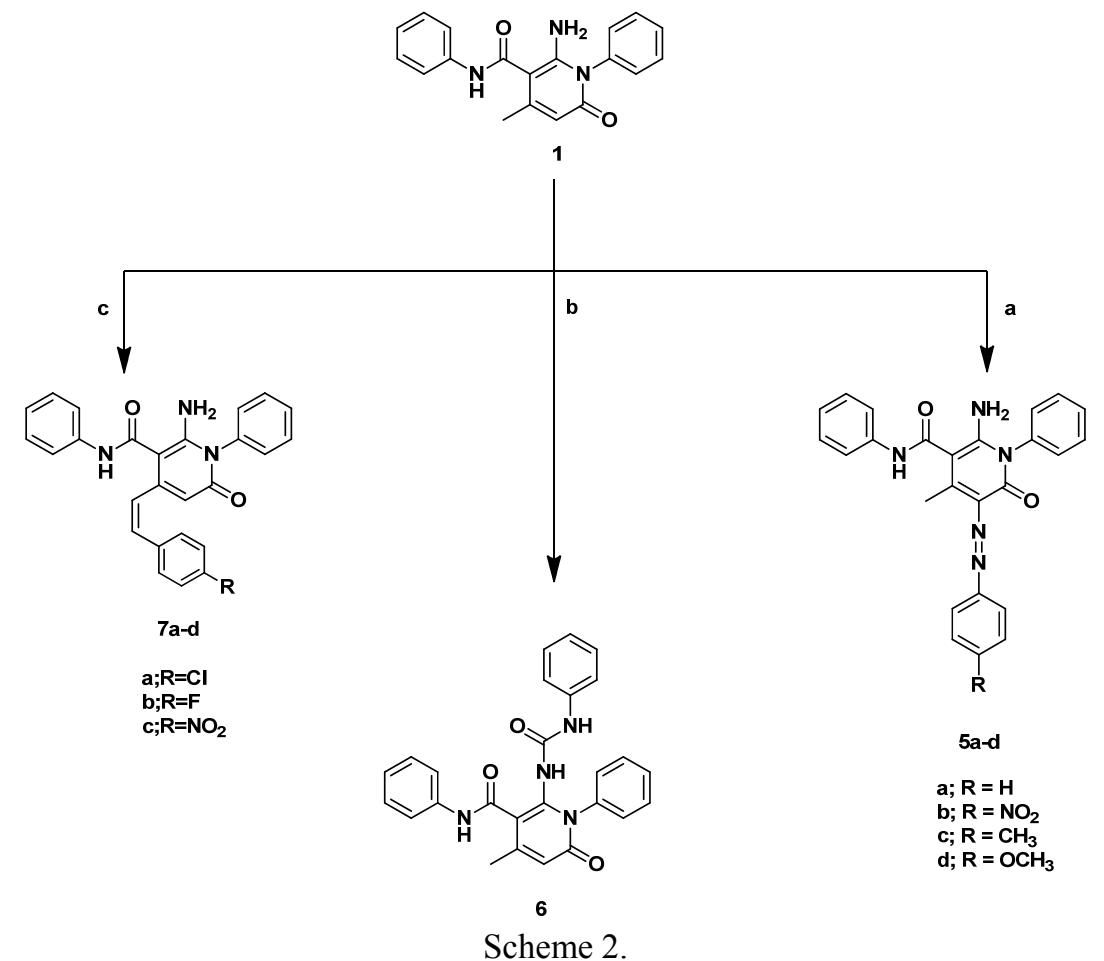

Reagents and conditions: a) $\mathrm{ArN}_{2} \mathrm{Cl}$, ethanol, $\mathrm{Na}$ acetate, $0{ }^{\circ} \mathrm{C}$, stirring. b) $\mathrm{PhNCO}$, fusion. c) substituted aromatic aldehydes, ethanol, piperidine, reflux.

Scheme 2, describes the schematic steps carried out in the synthesis of derivatives 5a-d, 6, 7a-d. Compounds 5a-d were prepared by hybridization of the aryldiazenyl moiety with the pyridone ring through a hydrazine linker. Thus, diazodization of aromatic amines with hydrochloric acid and sodium nitrite gave the diazonium salts, which were subsequently coupled with compound $\mathbf{1}$ in ethanolic sodium acetate to afford the target compounds (Hartz et al., 2010 \& Badea, Sxofei, Venterb \& Bercean, 2007).

The IR spectra of 5a-d showed the presence of characteristic absorption bands of $\mathrm{NH}, \mathrm{NH}_{2} .{ }^{1} \mathbf{H N M R}$ spectra of $\mathbf{5 b}$ and $\mathbf{5 c}$ as representative examples of these series revealed the existence of two doublets representing AB system at $\delta 7.68,8.25$ ppm and at $\delta 7.60,7.68 \mathrm{ppm}$ respectively with $J$ constant $=9 \mathrm{~Hz}$ for both of them, while ${ }^{1} \mathbf{H N M R}$ spectrum of $\mathbf{5 d}$ showed a singlet at $\delta 3.76 \mathrm{ppm}$ representing $\mathrm{OCH}_{3}$ protons. Moreover, ${ }^{13} \mathbf{C N M R}$ spectrum of $\mathbf{5 d}$ illustrated a characteristic signal at $55.31 \mathrm{ppm}$ corresponding to carbon of $\mathrm{OCH}_{3}$ group, in addition to a characteristic signal at $159.00 \mathrm{ppm}$ assigned to carbon of carbonyl amide and another one at $156.00 \mathrm{ppm}$ assigned to carbon of pyridone carbonyl. The title compound $\mathbf{6}$ was prepared through the fusion of compound $\mathbf{1}$ with slight excess of phenylisocyanate. The spectral data of compound $\mathbf{6}$ was in agreement with the assigned structure. IR spectrum displayed three bands at 3325, 3286, $3193 \mathrm{~cm}^{-1}$ corresponding to $3 \mathrm{NH}$ groups. Compounds $\mathbf{7 a - c}$ were prepared by refluxing compound $\mathbf{1}$ with an equimolar amount of substituted aromatic aldehydes in the presence of piperidine using absolute ethanol as a solvent (Ammar et al., 2004; Abdelghani, Shehab, El-Mobayed \& Abdel Hamid, 2012 and El-Sharkawy \& Ibrahim, 2013).

IR spectra of these derivatives showed the usual bands of $\mathrm{NH}, \mathrm{NH}_{2}$ at $3349,3264 \mathrm{~cm}^{-1}$ and carbonyl groups at 1655,1624 $\mathrm{cm}^{-1}$. ${ }^{1} \mathbf{H N M R}$ spectrum of $7 \mathbf{c}$ as a representative example of this group displayed two doublets representing AB system of the incorporated aldehyde at $\delta 7.68$ and $8.27 \mathrm{ppm}$ with $J$ constant $=8.4 \mathrm{~Hz}$. 


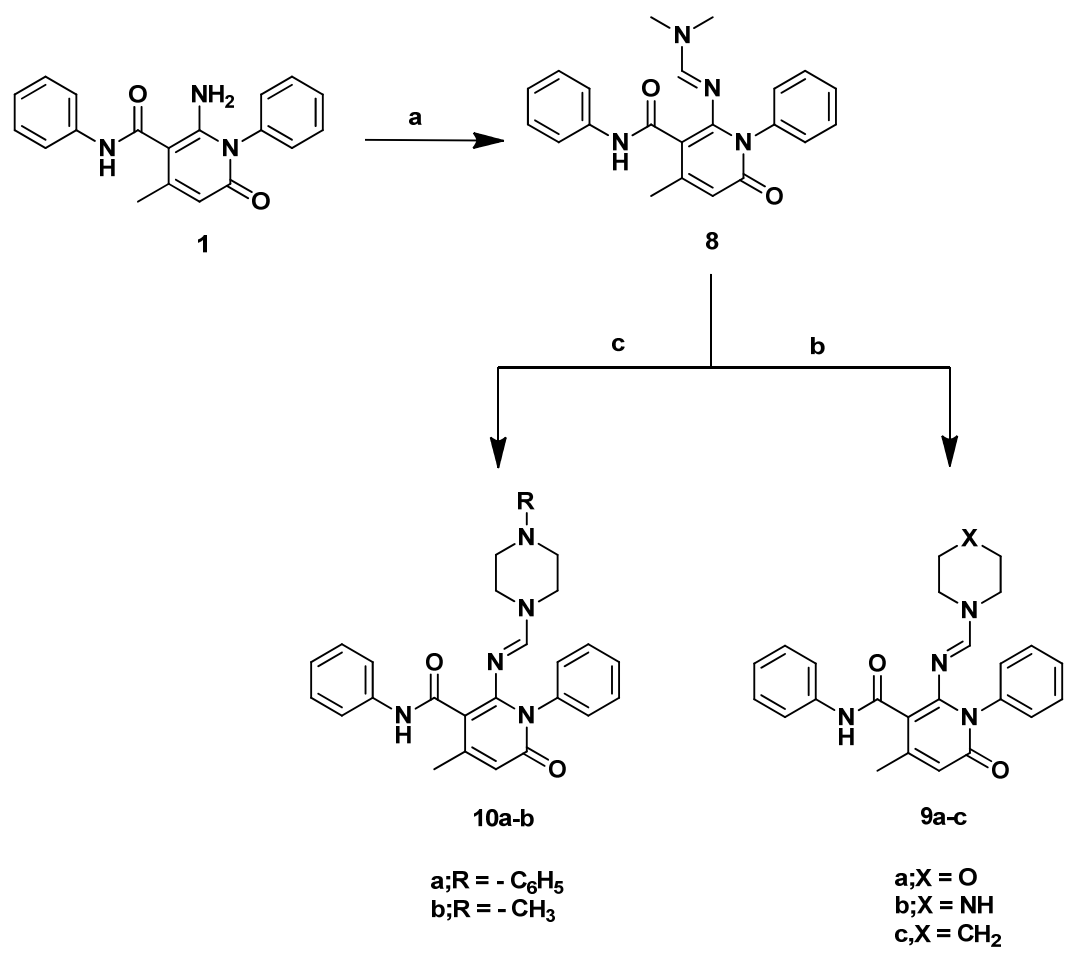

Scheme 3.

Reagents and conditions: a) DMF-DMA, dry xylene, reflux . b) secondary amines: (morpholine, piperazine and piperidine), ethanol, reflux .c) phenyl piperazine, methyl piperazine, ethanol, reflux.

The synthetic route followed for the preparation of compounds $\mathbf{9 a - c}$ and 10a,b is described in Scheme 3. Condensation of the amino group with DMF-DMA is one of the synthetic approaches to the synthesis of enamines. Thus, compound $\mathbf{8}$ was successfully prepared by refluxing compound 1 with DMF-DMA in dry xylene (Elneairy, Gad-Elkareem \& Taha, 2007). IR spectrum revealed sharp band at $3327 \mathrm{~cm}^{-1}$ corresponding to $\mathrm{NH}$ group, in addition to the disappearance of $\mathrm{NH}_{2}$ bands. ${ }^{1}$ HNMR displayed two singlets at $\delta 3.17$ and $3.24 \mathrm{ppm}$ representing the 6 protons of dimethylamino group, in addition to the singlet at $\delta 7.81 \mathrm{ppm}$ which is attributed to the imine proton. Furthermore, this derivative was used as an intermediate in the preparation of compounds 9a-c and 10a,b. Where, compounds 9a-c and 10a,b were successfully prepared according to the literature procedures via the reaction of the enamine $\mathbf{8}$ with an equimolar amount of appropriate secondary amines for 9a-c and substituted secondary amines for 10a,b in ethanol (Shawali, 2012 \& Al-Zaydia, Al-Shamarya \& Elnagdi, 2006).

The above mentioned structures were confirmed based on elemental and spectral data. Their structures were established by ${ }^{1}$ HNMR spectra which displayed the disappearance of the characteristic signals of dimethylamino group, along with the appearance of a singlet at $\delta 7.93-8.69 \mathrm{ppm}$ for the imine proton. Further confirmation was obtained by the MS spectra, which were consistent with their molecular weights. In addition, ${ }^{13} \mathbf{C N M R}$ spectrum of $10 \mathrm{~b}$ showed a characteristic signal at $35.91 \mathrm{ppm}$ assigned to carbon of $\mathrm{CH}_{3}-\mathrm{N}$ and at $65.98,66.26 \mathrm{ppm}$ corresponding to the four piperazine carbons, in addition to a characteristic signal at $157.14 \mathrm{ppm}$ assigned to imine carbon $\mathrm{N}=\underline{\mathrm{C}} \mathrm{H}-\mathrm{N}$ and 2 carbonyl groups at $\delta 164.80$ ppm.

\subsection{Anti-microbial Activity}

Antibacterial and antifungal activities of the newly synthesized compounds were performed at the Regional Center for Mycology and Biotechnology (RCMB), Al-Azhar University, Cairo, Egypt. Initially, target compounds 1, 2a-f, 3a-c, 4a,b, 5a-d, 6, 7a-c, 8, 9a-c, 10a,bandreference drugs were evaluated in-vitro for their antimicrobial and antifungal activities, using the broth microdilution reference method and minimum inhibitory concentration (MIC). The compounds were tested against two fungal strains (Aspergillus fumigates and Candida albicans), two Gram-positive bacteria (Bacillus subtillus and Streptococcus pneumonia) and two Gram-negative bacteria (Escherichia coli and Salmonella Typhimurium).

3.2.1 Anti-fungal Activity

Aspergillus fumigatusis largely responsible for increased the incidence of invasive aspergillosis (IA) in immunocompromised patients (Moore, Walls \& Denning, 2001). Thus, the activity of the novel compounds were 
presented in Table 1 and Table 2 which revealed that compounds $\mathbf{3 a}, \mathbf{5 c}$ and $\mathbf{9 b}$ were equipotent to the reference drug, Amphotericin B, against Aspergillus fumigatus ( $\mathrm{MIC}=1.95 \mu \mathrm{g} / \mathrm{ml}$ ), additionally, compound 3a was also equipotent to the reference drug against Candida albicans. Good activity was also observed for compounds 2 d, 3b, 5b and $9 \mathbf{a}$, against Aspergillus fumigatus (MIC $=3.9 \mu \mathrm{g} / \mathrm{ml}$ )

Table 1. The in-vitro antimicrobial activities, means of inhibitory $\% \pm$ Standard deviation produced on a range of clinically pathogenic microorganisms using $(125 \mu \mathrm{g})$ concentration of tested samples.

\begin{tabular}{|c|c|c|c|c|c|c|}
\hline \multirow{2}{*}{ Test Organism } & \multicolumn{2}{|l|}{ Fungi } & \multicolumn{2}{|c|}{ Gm +ve Bacteria } & \multicolumn{2}{|l|}{ Gm -ve Bacteria } \\
\hline & $\begin{array}{l}\text { Aspergillus } \\
\text { fumigatus }\end{array}$ & Candida albicans & $\begin{array}{l}\text { Streptococcus } \\
\text { pneumonia }\end{array}$ & Bacillis subtilis & Escherichia coli & $\begin{array}{l}\text { Salmonella } \\
\text { Typhimurium }\end{array}$ \\
\hline 1 & $16.15 \pm 0.25$ & $13.24 \pm 0.25$ & $14.38 \pm 0.58$ & $15.15 \pm 0.58$ & $14.16 \pm 0.44$ & $22.3 \pm 0.58$ \\
\hline $2 \mathrm{a}$ & $44.33 \pm 0.63$ & $32.25 \pm 0.44$ & $56.25 \pm 0.25$ & $67.25 \pm 0.37$ & $20.44 \pm 0.37$ & $32.15 \pm 0.63$ \\
\hline $2 b$ & $73.25 \pm 0.58$ & $60.44 \pm 0.63$ & $61.23 \pm 0.25$ & $71.24 \pm 0.25$ & $42.51 \pm 0.63$ & $51.24 \pm 0.58$ \\
\hline $2 c$ & $26.43 \pm 0.44$ & $20.63 \pm 0.63$ & $28.14 \pm 0.37$ & $35.3 \pm 0.25$ & $19.25 \pm 0.37$ & $29.8 \pm 0.58$ \\
\hline $2 d$ & $80.63 \pm 0.58$ & $72.41 \pm 0.37$ & $69.21 \pm 0.44$ & $81.32 \pm 0.58$ & $62.14 \pm 0.44$ & $72.1 \pm 0.58$ \\
\hline $2 \mathrm{e}$ & NA & NA & NA & NA & NA & NA \\
\hline $2 \mathrm{f}$ & NA & NA & NA & NA & NA & NA \\
\hline $3 a$ & $86.24 \pm 0.28$ & $90.24 \pm 0.21$ & $72.32 \pm 0.12$ & $91.85 \pm 0.33$ & $66.34 \pm 0.19$ & $74.32 \pm 0.58$ \\
\hline $3 b$ & $76.25 \pm 0.24$ & $84.32 \pm 0.42$ & $71.21 \pm 0.39$ & $86.24 \pm 0.58$ & $71.24 \pm 0.44$ & $83.24 \pm 0.63$ \\
\hline $3 c$ & $72.61 \pm 0.24$ & $80.23 \pm 0.42$ & $69.25 \pm 0.44$ & $84.32 \pm 0.58$ & $68.32 \pm 0.44$ & $74.32 \pm 0.58$ \\
\hline $4 a$ & NA & NA & NA & NA & NA & NA \\
\hline $4 \mathrm{~b}$ & NA & NA & NA & NA & NA & NA \\
\hline Ampicillin & - & - & $86.32 \pm 0.58$ & $99.62 \pm 0.63$ & - & - \\
\hline Gentamicine & - & - & - & - & $75.42 \pm 0.58$ & $86.32 \pm 0.63$ \\
\hline $\begin{array}{l}\text { Amphotericin } \\
\text { B }\end{array}$ & $90.31 \pm 0.58$ & $95.21 \pm 0.44$ & - & - & - & - \\
\hline
\end{tabular}

Table 2. Antimicrobial activity as MICs $(\mu \mathrm{g} / \mathrm{ml})$ of tested samples against tested organisms

\begin{tabular}{|c|c|c|c|c|c|c|}
\hline \multirow[t]{2}{*}{ Test Organism } & \multicolumn{2}{|c|}{ Fungi } & \multicolumn{2}{|c|}{ Gm +ve Bacteria } & \multicolumn{2}{|c|}{ Gm -ve Bacteria } \\
\hline & $\begin{array}{l}\text { Aspergillus } \\
\text { fumigatus }\end{array}$ & $\begin{array}{l}\text { Candida } \\
\text { albicans }\end{array}$ & $\begin{array}{l}\text { Streptococcus } \\
\text { pneumonia }\end{array}$ & Bacillis subtilis & Escherichia coli & $\begin{array}{c}\text { Salmonella } \\
\text { Typhimurium }\end{array}$ \\
\hline \multicolumn{7}{|l|}{ Comp. No. } \\
\hline 1 & 125 & 125 & 125 & 125 & 125 & 125 \\
\hline $2 b$ & 7.81 & 15.63 & 15.63 & 7.81 & 31.25 & 31.25 \\
\hline $2 d$ & 3.9 & 7.81 & 7.81 & 3.9 & 15.63 & 7.81 \\
\hline $3 a$ & 1.95 & 1.95 & 7.81 & 1.95 & 7.81 & 7.81 \\
\hline $3 b$ & 3.9 & 3.9 & 7.81 & 1.95 & 7.81 & 3.9 \\
\hline $3 c$ & 7.81 & 3.9 & 7.81 & 3.9 & 7.81 & 7.81 \\
\hline $5 a$ & 15.63 & 31.25 & 7.81 & 3.9 & 31.25 & 7.81 \\
\hline $5 b$ & 3.9 & 15.63 & 1.95 & 1.95 & 15.63 & 3.9 \\
\hline $5 c$ & 1.95 & 7.81 & 1.95 & 0.98 & 7.81 & 1.95 \\
\hline $5 d$ & 62.5 & 31.25 & 31.25 & 15.63 & 31.25 & 15.63 \\
\hline $9 a$ & 3.9 & 3.9 & 3.9 & 0.98 & 15.63 & 3.9 \\
\hline $9 b$ & 1.95 & 7.81 & 7.81 & 3.9 & 31.25 & 7.81 \\
\hline Ampicillin & - & - & 1.95 & 0.98 & - & - \\
\hline Gentamicine & - & - & - & - & 7.81 & 1.95 \\
\hline Amphotericin B & 1.95 & 1.95 & - & - & - & - \\
\hline
\end{tabular}

3.2.2 Anti-bacterial Activity

The obtained results against Gram-positive and Gram-negative bacteria showed that all the tested compounds exhibited superior activity to the starting 2-amino-4-methyl-6-oxo-N,1-diphenyl-1,6-dihydropyridine-3-carboxamide $\mathbf{1}$. Interestingly, compound $\mathbf{5 c}$ displayed broad-spectrum antibacterial activity equipotent to both Ampicillin and Gentamicin against the tested bacteria. This result shows that the introduction of the aryldiazenyl moiety in position 5 greatly enhanced the antibacterial activity. Moreover, in this series the 4-methylphenyl showed better activity than the nitro- and methoxyhpenyl counterparts with the exception of $\mathbf{5 b}$ which was equipotent to Ampicillin against the gram-positive bacteria, Streptococcus pneumonia. Additionally, the incorporation of morpholinomethylene to the 2-amino group resulted in an improved activity against Bacillus subtillus as demonstrated by compound 9a, MIC value $=0.98 \mu \mathrm{g} / \mathrm{mL}$, equivalent to Ampicillin. Interestingly, the 2-benzamido derivatives 3a-c exhibited good activity against Escherichia coli with same potency as Gentamicin, $7.81 \mu \mathrm{g} / \mathrm{mL}$, these results show that acylation of the amino group directs the activity against this bacterial strain, it also shows that the activity is not affected by the substituents on the phenyl ring. All the tested compounds were inactive against the gram-negative bacteria, Pseudomonas aeruginosa. Moreover, compounds $\mathbf{2 e , f , 4 a , b}$ and $\mathbf{6}$ were devoid of any antifungal or antibacterial activities. This may suggest that 
reactions which transform the amino group to alkylamino or urea moieties were not beneficial for the activity contrary to the benzamido-substitution as compounds 3a,b. Furthermore, with the exception of compounds $\mathbf{2 d}$ and $\mathbf{9 a}$, the potency was greatly reduced in the enamine series such as compounds 2a-f, 9a-c and 10a,b.

\subsection{D QSAR Study}

\subsubsection{Development of QSAR Models}

QSAR analyses for antibacterial and antifungal activities of the prepared derivatives(1, 2b,d, 3a-c, 5a-d and 9a,b ) were performed in order to correlate these activities with thestructural features of the synthesized compounds, and to identify the positive and negative structural features within them.

The analysis was run by means of the DS 2.5 software (Discovery Studio 2.5, Accelrys, Co., Ltd., San Diego, CA, USA). A set of the newly synthesized 2-pyridones, twelve compounds, was used as a trainingset with their measured pMIC against Bacillis subtilis, Aspergillus fumigates and Escherichia coli for QSAR modeling. Compounds $(1, \mathbf{5 c}$ and 5d) were adopted as an external test subset for validating the QSAR models. "Calculate Molecular Properties" module was used for calculatingdifferent molecular properties for the training set compounds. 2D Descriptors involved: AlogP, molecular properties, molecular property counts, surface area and volume and topological descriptors, while the 3D descriptors involved: Dipole, jurs descriptors, principle moments of inertia, shadow indicesand surface area and volume. Genetic function approximation (GFA) was utilized to search for the best possible QSAR regression equation capable of correlating the variations in the biological activities ofthe training set compounds with variations in the generated descriptors, i.e., multiple linear regressionmodeling (MLR). QSAR model was validated employing leave one-out cross-validation by settingthe folds to a number much larger than the number of samples, $r^{2}$ (squared correlation coefficient value) and $r^{2}$ prediction (predictive squared correlation coefficient value), residuals between the predicted andexperimental activity of the test set and training set.

\subsubsection{QSAR Study Results}

Equation (1).Represents the best performing QSAR model for the activity against Aspergillus fumigates;

$-\log M I C=1.3196+0.3312$ Num_RotatableBonds -5.8276 Shadow_YZfrac (1)

Equation (2).Represents the best performing QSAR model for the activity against Bacillus subtillus;

$-\log$ MIC $=4.3021$ - 1.6266e-003 PMI_Z - 5.1754 Shadow_XYfrac (2)

Equation (3).Represents the best performing QSAR model for the Escherichia coli;

$-\log \mathrm{MIC}=-1.2398-7.1752 \mathrm{e}-002$ ES_Sum_dsN $-8.1325 \mathrm{e}-004$ Jurs_WNSA_2 (3)

According to equations (1)-(3), the QSAR models were represented graphically by scattering plotsof the experimental versus the predicted bioactivity values $-\log$ MIC for the training set compounds asshown in Figures 2-4. The method used to build the model was Least-Squares, $r^{2}=0.75,0.818$ and 0.818 , respectively, $r^{2}$ (adj) $=0.667,0.766$ and 0.766 , respectively, $r^{2}$ (pred) $=0.404,0.672$ and 0.635 ,respectively, Least-Squared error $=0.019,0.022$ and 0.0113 , respectively, where $r^{2}$ (adj) is $r^{2}$ adjustedfor the number of terms in the model; $r^{2}$ (pred) is the prediction $c$, equivalent to $q^{2}$ from a leave-1-outcross validation.

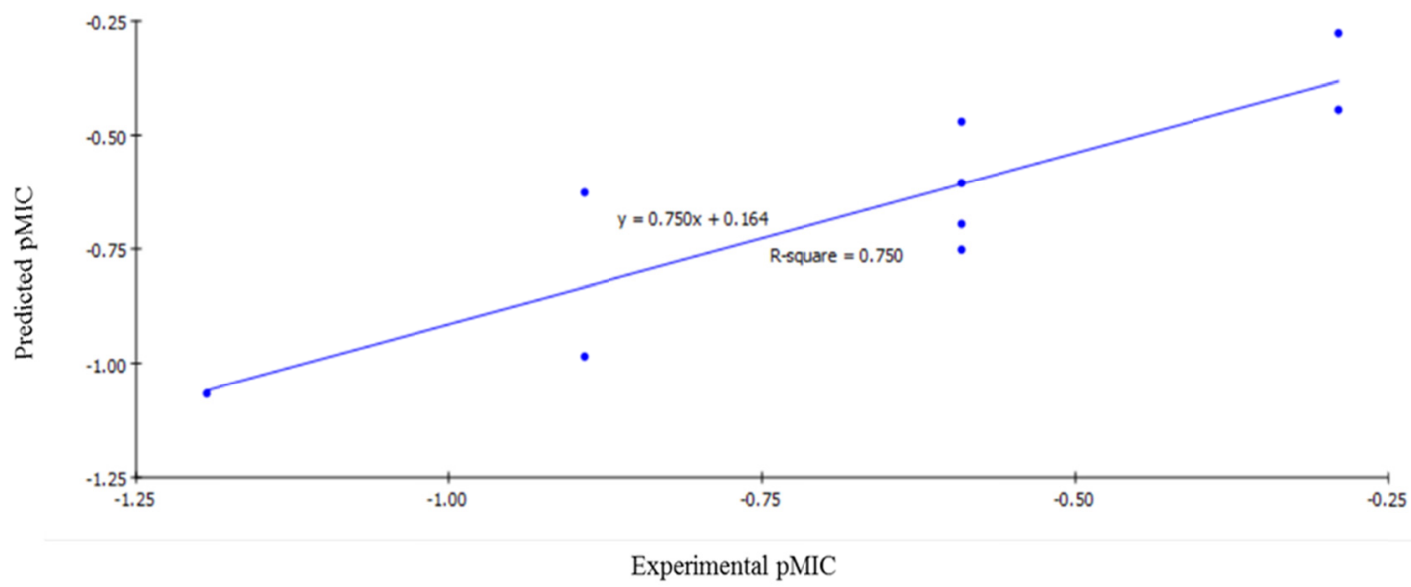

Figure 2. Predicted versus experimental pMIC of the tested compounds against Aspergillus fumigates according to equation (1), $\mathrm{r}^{2}=0.750$. 


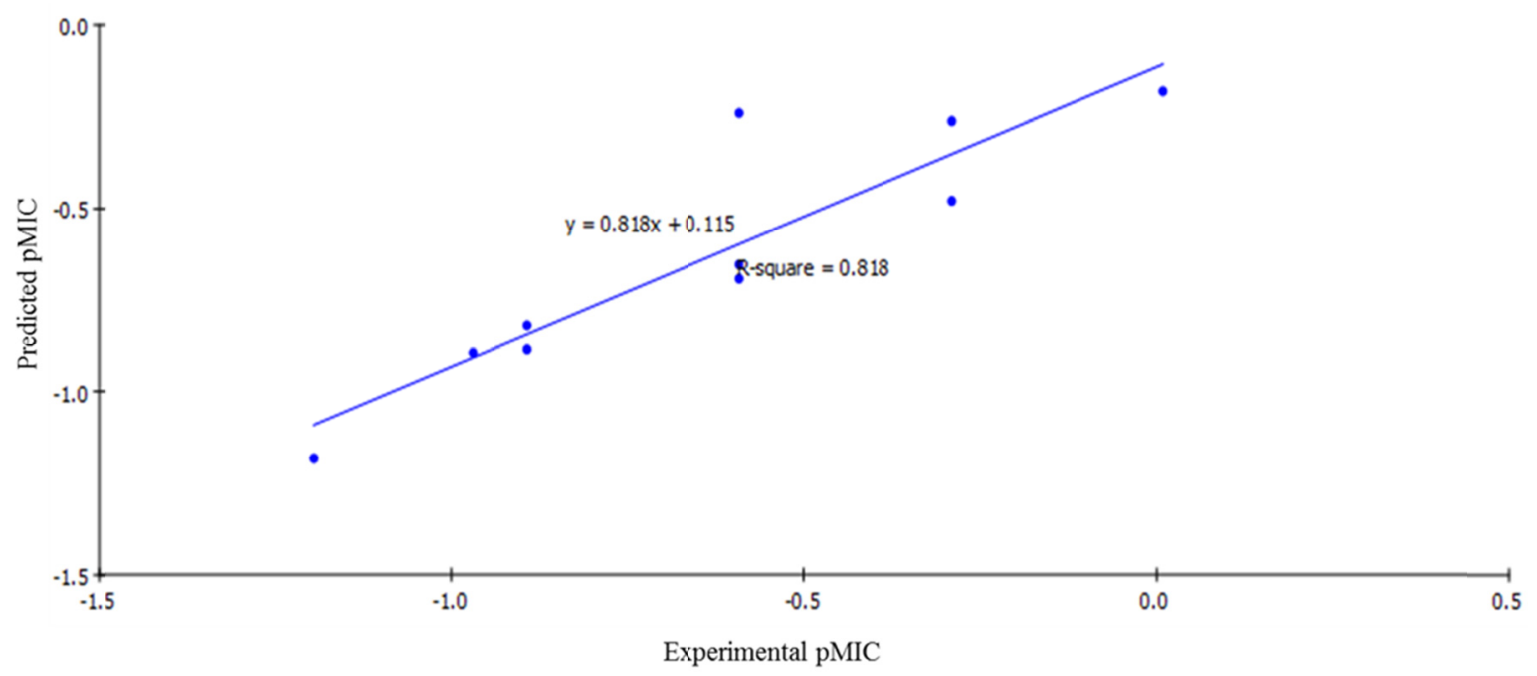

Figure 3. Predicted versus experimental pMIC of the tested compounds against Bacillus subtillus according to equation $(2), \mathrm{r} 2=0.818$.

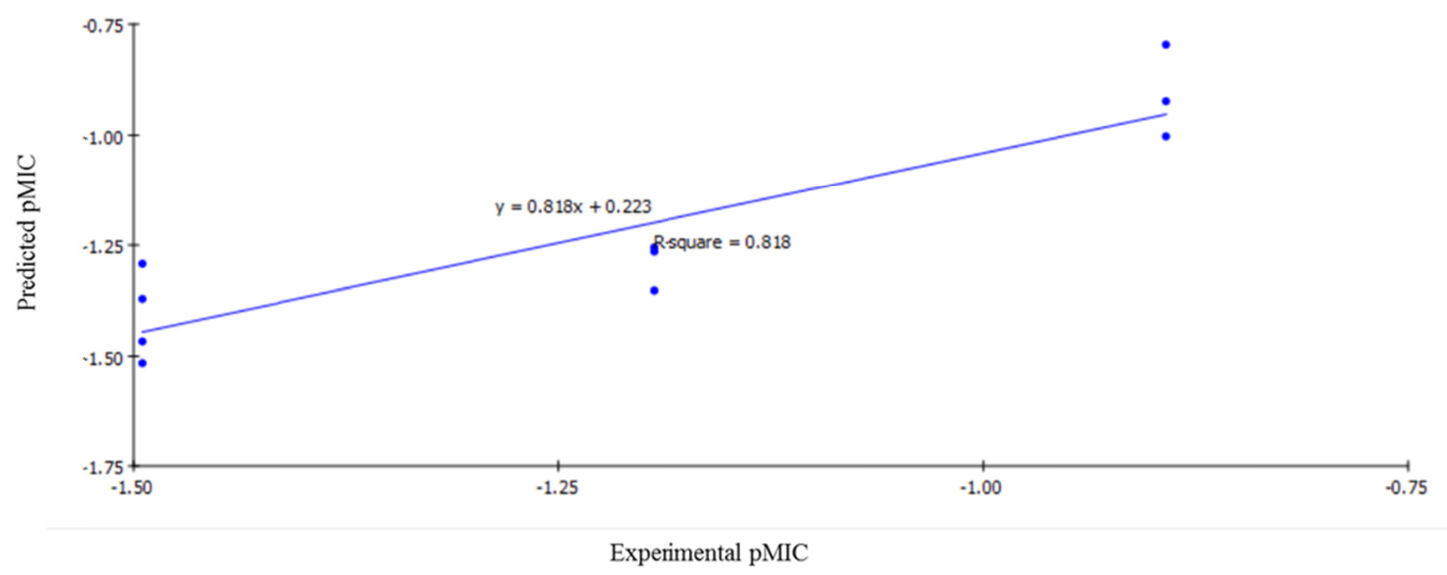

Figure 4. Predicted versus experimental PMIC of the tested compounds against Escherichia coli according to equation (3), $\mathrm{r} 2=0.818$.

In conclusion, equation (1) suggested that the antifungal activity of the synthesized compounds is mainly affected by molecular property counts (Num_RotatableBonds) and shadow index, Shadow_YZfrac. Rotatable bonds, are defined as single bonds between heavy atoms that are both not in a ring and not terminal, i.e., connected to a heavy atom that is attatched to only hydrogens. While shadow indices are a set of geometric descriptors to characterize the shape of the molecules (Rohrbaug, 1987). The descriptors are calculated by projecting the model surface on three mutually perpendicular planes: $x y, y z$, and $x z$. These descriptors depend not only on conformation, but also on the orientation of the model. To calculate them, the models are first rotated to align the principal moments of inertia with the $x$-, $y$-, and $z$-axes. Shadow_YZ is area of the molecular shadow in the $y z$ plane. On the other hand, equation (2) indicated that the principal moments of inertia-Z component (PMI_Z) and the shadow index (Shadow_XYfrac) were the principle descriptors contributing negatively to the activity of the compounds against Bacillus subtillus. Whereas, the principal moments of inertia calculates the principal moments of inertia about the principal axes of a molecule according to certain rules: the moments of inertia are computed for a series of straight lines through the center of mass (Hill, 1960). The moments of inertia are given by: distances are established along each line proportional to the reciprocal of the square root of $I$ on either side of the center of mass. The locus of these distances forms an ellipsoidal surface. The principal moments are associated with the principal axes of the ellipsoid. If all three moments are equal, the molecule is considered to be a symmetrical top. If no moments are equal, the molecule is considered to be an unsymmetrical top. Finally, equation (3) shows that the activity against Escherichia colimight be affected by the E-state sum for nitrogen atom (ES_Sum_dsN) and Jurs descriptor (Jurs_WNSA_2). The estate keys calculate the sums of electrotopological state (E-state) values and/or the counts of each atom type (Hall \& kier, 2000) \& Hall, Mohney \& kier, 1991). ES_Sum_dsN calculates the E-state count for nitrogen. Moreover, Jurs descriptors are those ones that combine shape and electronic 
information to characterize molecules (Rohrbaugh, 1987).The descriptors are calculated by mapping atomic partial charges on solvent-accessible surface areas of individual atoms. Jurs_WNSA_2 is surface-weighted charged partial surface areas, it is calculates by multiplying a set of six descriptors (partial positive surface area, partial negative surface area, total charge weighted negative surface area, total charge weighted positive surface area, atomic charge weighted positive surface area, atomic charge weighted negative surface area) the total molecular solvent-accessible surface area and dividing by 1000 .

\subsubsection{QSAR Validation}

Robustness of the established QSAR models (1, 2 and 3) was verified by using; Leave-one-out (LOO) internal validation $\left(r^{2}=0.75,0.818\right.$ and 0.818 , respectively). Cross-validation was also employed where $q^{2}$, which is equivalent to $r^{2}$ (pred), was $0.404,0.672$ and 0.635 , respectively. In addition, validation was employed by measuring the residuals between the experimental and the predicted activities of the training set (Table 3 ).

Table 3. Experimental activities of the synthesized derivatives against the predicted activities according to equations 1,2 and 3 .

\begin{tabular}{|c|c|c|c|c|c|c|c|c|c|}
\hline Comp & $A s p$ & ergillus fumi & & & acillus subti & & & scherichia co & \\
\hline & $\begin{array}{l}\text { Experimental } \\
\text { Activity } \\
\text { (pMIC) }\end{array}$ & $\begin{array}{l}\text { Predicted } \\
\text { Activity } \\
\text { (pMIC) }\end{array}$ & Residuals & $\begin{array}{l}\text { Experimental } \\
\text { Activity } \\
\text { (pMIC) }\end{array}$ & $\begin{array}{l}\text { Predicted } \\
\text { Activity } \\
\text { (pMIC) }\end{array}$ & Residuals & $\begin{array}{l}\text { Experimental } \\
\text { Activity } \\
\text { (pMIC) }\end{array}$ & $\begin{array}{c}\text { Predicted } \\
\text { Activity } \\
\text { (pMIC) }\end{array}$ & Residuals \\
\hline $2 b$ & -0.892095 & -0.695721 & 0.0930167 & -0.892651 & -0.885362 & -0.00728928 & -1.49485 & -1.29206 & -0.202789 \\
\hline $2 d$ & -0.591065 & -0.695721 & 0.104656 & -0.892651 & -0.820696 & -0.071955 & -1.19396 & -1.26466 & 0.0707032 \\
\hline $3 a$ & -0.290035 & -0.444128 & 0.154093 & -0.290035 & -0.261903 & -0.0281316 & -0.892651 & -1.00249 & 0.109841 \\
\hline $3 b$ & -0.591065 & -0.47012 & -0.120945 & -0.290035 & -0.47968 & 0.189645 & -0.892651 & -0.923027 & 0.0303755 \\
\hline $3 c$ & -0.892095 & -0.626313 & -0.265782 & -0.591065 & -0.692944 & 0.101879 & -0.892651 & -0.796406 & -0.096245 \\
\hline $5 \mathrm{a}$ & -1.19396 & -1.06652 & -0.127436 & -0.591065 & -0.653966 & 0.0629012 & -1.49485 & -1.51574 & 0.0208936 \\
\hline $5 b$ & -0.591065 & -0.752191 & 0.161126 & -0.969416 & -0.894512 & -0.074904 & -1.19396 & -1.25509 & 0.0611264 \\
\hline $5 c$ & ---------- & ------- & ------ & 0.00877392 & -0.180768 & 0.189541 & ------- & ------ & --------- \\
\hline $5 \mathrm{~d}$ & -------- & ------- & ----- & --------- & ------- & -------- & -1.49485 & -1.46673 & -0.0281184 \\
\hline $9 a$ & -0.591065 & -0.605672 & 0.0146066 & 0.00877392 & -0.180768 & 0.189541 & -1.19396 & -1.3522 & 0.158237 \\
\hline $9 b$ & -0.290035 & -0.276699 & -0.0133358 & -0.591065 & -0.240204 & -0.350861 & -1.49485 & -1.37083 & -0.124024 \\
\hline
\end{tabular}

Moreover, the experimental and expected activities as well as the residuals of the compounds, used as statistical outliers in building the three models, are presented in (Table 4). Interestingly, the predicted activities by the generated QSAR models were very close to those observed experimentally, indicating that these models could be applied for further prediction of more effective hits having the same skeletal framework.

Table 4. Experimental activities of compounds $1,5 \mathrm{c}$ and $5 \mathrm{~d}$, used as statistical outliers against the predicted activities according to equations 1,2 and 3 .

\begin{tabular}{|c|c|c|c|c|c|c|c|c|c|}
\hline \multirow[t]{4}{*}{ Comp } & \multicolumn{3}{|c|}{ Aspergillus fumigatus } & \multicolumn{3}{|c|}{ Bacillus subtillus } & \multicolumn{3}{|c|}{ Escherichia coli } \\
\hline & Experimental & Predicted & Residuals & Experimental & Predicted & Residuals & Experimental & Predicted & Residuals \\
\hline & Activity & Activity & & Activity & Activity & & Activity & Activity & \\
\hline & (pMIC) & (pMIC) & & (pMIC) & (pMIC) & & (pMIC) & (pMIC) & \\
\hline 1 & -2.09691 & -2.09691 & $-4.0496 \mathrm{e}-12$ & -2.09691 & -2.09691 & $-3.59579 \mathrm{e}-12$ & -2.09691 & -2.09691 & $-4.9378 \mathrm{e}-12$ \\
\hline $5 \mathrm{c}$ & -0.290035 & -0.290035 & $1.03528 \mathrm{e}-11$ & ------- & --------- & ---------- & -0.290035 & -0.290035 & $3.60278 \mathrm{e}-12$ \\
\hline $5 \mathrm{~d}$ & -1.79588 & -1.79588 & $-7.1334 \mathrm{e}-12$ & -0.290035 & -0.261903 & -0.0281316 & -------- & ---------- & -------- \\
\hline
\end{tabular}

\section{Conclusions}

In the present work twenty six novel derivatives of 6-oxo-pyridine-3-carboxamide were synthesized and evaluated as antibacterial and antifungal agents, using Amphotericin B, Ampicillin and Gentamicin as reference drugs. Amongst these novel compound, the 5-(p-tolyldiazenyl)-1,6-dihydropyridine-3-carboxamide derivative, $\mathbf{5 c}$, displayed broad-spectrum antibacterial activity equipotent to both Ampicillin and Gentamicin against the tested bacteria. It also showed antifungal activity comparable to Amphotericin B. The 2D QSAR models generated by Discovery studio 2.5 software, showed some important geometric and molecular descriptors that might be controlling the activities of these novel compounds. These results suggest that the novel pyridone derivatives could be further investigated for their potential antifungal and antibacterial activities. 


\section{References}

Abdelghani, E., Shehab, W. S., El-Mobayed, M., \& Abdel Hamid, A. M. (2012). Genetic and Biochemical Characterization of Unsaturated Fatty Acids of Yeast Strains, Int. J. Basic. Appl. Sci., 1, 220-236.

Alekshun, M. N., \& Levy, S. B. (2007). Cell, 128, 1037-1050. http://dx.doi.org/10.1016/j.cell.2007.03.004

Al-Zaydia, K. M., Al-Shamarya, A., \& Elnagdi, M. H. (2006). Molecular Mechanisms of Antibacterial Multidrug Resistance, J. Chem. Res., 6, 408-411.

Ammar, Y. A., El-Sharief, A. M., Al-Sehemi, A. G., Mohamed, Y. A., Senussia, M. A., \& El-Gaby. M. S. (2005). Cyanoacetanilides Intermediates in Heterocyclic Synthesis. Part 2: Preparation of Some Hitherto Unknown Ketene Dithioacetal, Benzoazole and Pyridone Derivatives, J. Chinese. Chem. Soc., 52, 553-558. http://dx.doi.org/10.1002/jccs.200500081

Ammar, Y. A., El-Sharief, A. M., Mohamed, Y. A., Salem, M. A., Al-Sehemi, A. G., \& El-Gaby, M. S. (2004). Treatment of o-Alkynylphenylsydnones with Acids: A New Route to 3-Arylcinnolines, J Chinese Chem. Soc., 51, 975-981. http://dx.doi.org/10.1002/jccs.200400145

Badea, V., Sxofei, M. D., Venterb, M. M., \& Bercean, V. N. (2007). Regioselective Alkylation of 1H-7-ethoxycarbonyl-6-methyl-3-phenyl-pyrazolo[5,1-c][1,2,4]triazole and 1H-6-methyl-3-phenyl-pyrazolo [5,1-c][1,2,4]triazole, Tetrahedron, 63, 1467-1473. http://dx.doi.org/10.1016/j.tet.2006.11.067

Bax, R., Mullan, N., \& Verhuef, J. (2000). The Millennium Bugs - the Need For and Development of New Antibacterials, Int. J. Antimicrob. Agents, 16, 51-59. http://dx.doi.org/10.1016/S0924-8579(00)00189-8

Cohen, M. L. (1992). Epidemiology of Drug Resistance: Implications for a Post-Antimicrobial Era, Science 257, 1050-1055. http://dx.doi.org/10.1126/science.257.5073.1050

Elneairy, M. A., Gad-Elkareem, M. A., \& Taha, A. M. (2007). Reactions with Dimethylformamide-dimethylacetal: Synthesis and Reactions of Several New Pyridine and pyrazolo[3,4-b]pyridine Derivatives, Heteroatom Chem., 18 , 399-404. http://dx.doi.org/10.1002/hc.20312

El-Sharkawy, K. A., \& Ibrahim, R. A. (2013). New Approaches For The Synthesis And Antitumor Evaluation Of Pyridine, Thieno 3, 4-c Pyridine, Pyrazolo 3, 4-b Pyridine And Pyrido 3, 4-d Pyridazine Derivatives, Eur. Chem. Bull., 2, 530-537.

Fadda, A. A., Bondock, S., Rabie, R., \& Etman, H. A. (2008). Cyanoacetamide Derivatives as Synthons in Heterocyclic Synthesis, Turk. J. Chem., 32, 259-286.

Finch, R., Greenwood, D., Norrby, S. R., \& Whitley, R. J. (2003). Antibiotic and Chemotherapy: Anti-infective Agents and Their Use in Therapy, J. Antimicrob. Chemother., 52(4), 740-741. http://dx.doi.org/10.1093/jac/dkg432

Gerusz, V. (2010). Annual Reports in Med. Chem., 45, 295-311. http://dx.doi.org/10.1016/S0065-7743(10)45018-6

Gupta, A. K., \& Plott, T. (2004). Ciclopirox for the Treatment of Superficial Fungal Infections: A Review, Int. J. Dermatol., 3, 43-49. PMID: 12895181

Hall, L. H., \& Kier, L. B. (2000). The E-State as the Basis for Molecular Structure Space Definition and Structure Similarity, J. Chem. Inform. Comput. Sci., 40, 784-791. http://dx.doi.org/10.1021/ci990140w

Hall, L. H., Mohney, B., \& Kier, L. B. (1991). The Electrotopological State: Structure Information at the Atomic Level for Molecular Graphs, J. Chem. Inform. Comput. Sci., 31, 76-82. http://dx.doi.org/10.1021/ci00001a012

Hartz, R. A., Ahuja, V. T., Schmitz, W. D., Molski, T. F., Mattson, G. K., Lodge, N. J., Bronson, J. J., \& Macor, J. E. (2010). Synthesis and Structure-Activity Relationships of $N^{3}$-pyridylpyrazinones as Corticotropin-releasing factor-1 $\left(\mathrm{CRF}_{1}\right) \quad$ receptor antagonists, Bioorg. Med. Chem. Lett., $20, \quad 1890-1894$. http://dx.doi.org/10.1016/j.bmcl.2010.01.129

Hill T. L. (1960). Moments of inertia: Introduction to statistical Thermodynamics, Addison-Wesley, Reading, MA., $1^{\text {st }}$ edition

Khokhani, K., Khatri, T., \& Patel, P. (2013). One Ppot Synthesis of Bioactive Novel Cyanopyridines, J. Korean Chem. Soc., 57(4), 476-482. http://dx.doi.org/10.5012/jkcs.2013.57.4.476

Kim, C. M., Kwak, J., Lee, C., Park, H., Kang, S., Kim, J., Song, Y., Ro, S., Lee, T., \& Cho. (2005). In Vitro Activities of CG400549, a Novel FabI Inhibitor, against Recently Isolated Clinical Staphylococcal Strains in Korea, J. abstr. F-1880, p. 218. Abstr. 45 ${ }^{\text {th }}$ Intersci. Conf. Antimicrob. Agents Chemother., American Society for Microbiology, Washington, DC 
Li, Q., Mitscher, L. A., \& Shen, L. L. (2000). The 2-Pyridone Antibacterial Agents: Bacterial Topoisomerase Inhibitors, Med. Res. Rev., 20, 231. http://dx.doi.org/10.1002/1098-1128(200007)20:4<231::AID-MED1>3.0.CO;2-N

Mohamed, M. S., Awad, Y. S., El-Hallouty, S. M., \& El-Araby, M. (2012). Design, Synthesis and Cancer Cell Line Activities of Pyrazolo[3,4-b]pyridine Derivatives, Open J. Med. Chem., 2, 78-88. http://dx.doi.org/10.4236/ojmc.2012.23010

Moore, C. B., Walls, C. M., \& Denning, D. W. (2001). In Vitro Activities of Terbinafine against Aspergillus Species in Comparison with Those of Itraconazole and Amphotericin B, Antimicrob. Agents Chemother., 45, 1882-1885. http://dx.doi.org/10.1128/AAC.45.6.1882-1885.2001

National Committee for Clinical Laboratory Standards. (2002). Methods for broth dilution antimicrobial susceptibility: approved standard M27-A2, $2^{\text {nd }}$ ed. National Committee for Clinical Laboratory Standards, Wayne, Pa.

Neu, H. C. (1992). The Crisis in Antibiotic Resistance, Science, 257, 1064-1073. http://dx.doi.org/10.1126/science.257.5073.1064

Park, H. S., Yoon, Y. M., Jung, S. J., Kim, C. M., Kim, J. M., \& Kwak. J. H. (2007). Antistaphylococcal activities of CG400549, a new bacterial enoyl-acyl carrier protein reductase (FabI) inhibitor, J. Antimicrob. Chemother., 60, 568-574. http://dx.doi.org/10.1093/jac/dkm236

Parmar, K. A., Prajapati, S. N., Joshi, S. A., Goswami, K. V., Patel, A. N. (2011). Studies on Cyanopyridones and Isoxazoles ring system in the synthesis of Novel bioactive compounds, Der Chemica Sinica, 2, 100-110.

Ritter, T. K. \& Wong, C. H. (2001). Carbohydrate-Based Antibiotics: A New Approach to Tackling the Problem of Resistance, Ang. Chem. Int., 35, 3508-3533. http://dx.doi.org/10.1002/1521-3773(20011001)40:19<3508::AID-ANIE3508>3.0.CO;2-I

Rohrbaugh, R. H. P. (1987). Descriptions of Molecular Shape Applied in Studies of Structure/Activity and Structure/Property Relationships, Anal, Chim. Acta., 199, 99-109.

Saiki, A. Y. C., Shen, L. L., Chen, C. M., Baranowski, J., \& Lerner, C. G. (1999). DNA Cleavage Activities of Staphylococcus aureus gyrase and Topoisomerase IV Stimulated by Quinolones and 2-Pyridones, Antimicrob. Agents Chemother., 43, 1574.

Schiebel, J., Chang, A., Shah, S., Lu, Y., Liu, L., Pan, P., Hirschbeck, M. W., Tareilus, M., Eltschkner, S., Yu, W., Cummings, J. E., Knudson, S. E., Bommineni, G. R., Walker, S. G., Slayden, R. A., Sotriffer, C. A., Tonge, P. J., \& Kisker. C. (2014). Rational Design of Broad Spectrum Aantibacterial Activity Bbased on a Clinically Relevant Enoyl-acyl Carrier Protein (ACP) Reductase Inhibitor, J. Biol. Chem., 289(23), 15987-16005. http://dx.doi.org/10.1074/jbc.M113.532804

Setti, E. L., Quattrocchio, L., \& Micetich, R. G. (1997). Current Approaches to Overcome Bacterial Resistance, Drugs Future, 22, 271-284.

Shawali, A. S. J. (2012). Site-selectivity in Hydrazinolysis of 3,4'-bis-(functionalized carbonyl)-4,3'-bis(pyrazolyl)ketones. A Convenient Synthesis of 4-(pyrazol-3-yl)-2H-pyrazolo[3,4-d]-pyridazines, Arkivoc, I, 383-43. http://dx.doi.org/10.3998/ark.5550190.0013.525

Struelens, M. J. (1998). The Epidemiology of Antimicrobial Resistance in Hhospital Acquired Infections: Problems and Possible Solutions, Br. Med. J., 317, 652-654. http://dx.doi.org/10.1136/bmj.317.7159.652

Tipparaju, S. K., Joyasawal, S., Forrester, S., Mulhearn, D. C., Pegan, S., Johnson, M. E., Mesecara, A. D., \& Kozikowski, A. P. (2008). Design and Synthesis of 2-Pyridones as Novel Inhibitors of the Bacillus anthracis enoyl-ACP Reductase, Bioorg. Med. Chem. Lett., 18, 3565-3569. http://dx.doi.org/10.1016/j.bmcl.2008.05.004

Vyas, D. H., Tala, S. D., Akbari, J. D., Dahduk, M. F., \& Joshi, H. S. (2008). Synthesis And Physicochemical Studies Of Some Molecules Of Medicinal Interest, Int. J. Synth. Characterization, 1(1), 103-107.

Yum, J. H., Kim, C., Yong, K. Lee, D. K., Chong, Y., Kim, C. M., Kim, J. M., Ro, S., \& Cho, J. M. (2007). In vitro Activities of CG400549, a Novel FabI Inhibitor, Against Recently Isolated Clinical Staphylococcal strains in Korea, Antimicrob. Agents Chemother, 51, 2591-2593. http://dx.doi.org/10.1128/AAC.01562-06

\section{Copyrights}

Copyright for this article is retained by the author(s), with first publication rights granted to the journal.

This is an open-access article distributed under the terms and conditions of the Creative Commons Attribution license (http://creativecommons.org/licenses/by/3.0/). 